\title{
Solar prominences: theory and models
}

\section{Fleshing out the magnetic skeleton}

\author{
Sarah E. Gibson ${ }^{1}$ \\ Received: 20 March 2018 / Accepted: 25 September 2018 / Published online: 22 October 2018 \\ (c) The Author(s) 2018
}

\begin{abstract}
Magnetic fields suspend the relatively cool material of solar prominences in an otherwise hot corona. A comprehensive understanding of solar prominences ultimately requires complex and dynamic models, constrained and validated by observations spanning the solar atmosphere. We obtain the core of this understanding from observations that give us information about the structure of the "magnetic skeleton" that supports and surrounds the prominence. Energetically-sophisticated magnetohydrodynamic simulations then add flesh and blood to the skeleton, demonstrating how a thermally varying plasma may pulse through to form the prominence, and how the plasma and magnetic fields dynamically interact.
\end{abstract}

Keywords Solar prominences · Magnetohydrodynamics · Corona: structures · Prominences: Magnetic fields · Prominences: Models

\section{Contents}

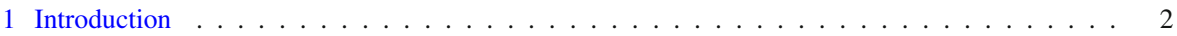

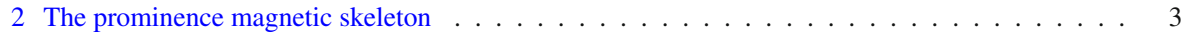

2.1 Prominence bones: magnetic dips . . . . . . . . . . . . . . . . . . . . 3

2.2 Early prominence models . . . . . . . . . . . . . . . . . . . . . . . . . . . . . . . .

2.3 Inverse versus normal magnetic fields . . . . . . . . . . . . . . . . . . . 6

2.4 Sheared-arcade versus flux-rope models . . . . . . . . . . . . . . . . . . . 7

2.4 Sheared-arcade models . . . . . . . . . . . . . . . . . . . . . . . 7

2.4 .2 Flux-rope models . . . . . . . . . . . . . . . . . . . . . . . 8

2.4.3 Comparing to prominence properties . . . . . . . . . . . . . . . . . 9

3 Beyond bones: the prominence in context . . . . . . . . . . . . . . . . . . . . 11

3.1 Filament channels and chirality . . . . . . . . . . . . . . . . . . . . . . . . . . . . . . . . . . . .

3.2 Sigmoids and separatrices . . . . . . . . . . . . . . . . . . . . . . . . 14

The National Center for Atmospheric Research is supported by the National Science Foundation.

Sarah E. Gibson

sgibson@ucar.edu

1 National Center for Atmospheric Research, 3080 Center Green Dr., Boulder, USA 
3.3 Cavities and flux surfaces . . . . . . . . . . . . . . . . . . . . . 16

4 Adding flesh and blood to the skeleton: incorporating dynamics and thermodynamics into prominence simulations . . . . . . . . . . . . . . . . . . . . . . 21

4.1 Prominence dynamics . . . . . . . . . . . . . . . . . . . . . . . 21

4.2 Prominence thermodynamics . . . . . . . . . . . . . . . . . . . . . . 24

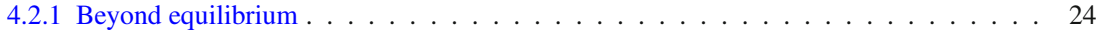

4.2 .2 Beyond force-free . . . . . . . . . . . . . . . . . . . . . . . . . . . . . . . . . 26

4.3 The Full Monty: prominence formation in 3D MHD . . . . . . . . . . . . . . . . . . . . . 27

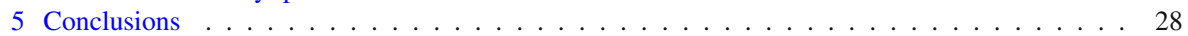

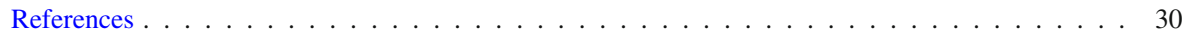

\section{Introduction}

Prominences are surprising. During a solar eclipse they are visible as dischordantly clumpy and bright (indeed, pink,) structures, sharply contrasting with the elegant pearly white streamers that make up most of the corona (Fig. 1). As we learn more about prominences through multiwavelength, high-resolution observations, the mysteries surrounding them only intensify. How can something that is relatively cool and dense be suspended in a hot and sparse atmosphere? Where do they come from, and how do they go?

The answers to these questions clearly involve magnetism, the controlling force of the solar corona. In particular, the abiding presence of a prominence implies a magnetic structure that can provide sustained gravitational support and thermal isolation. Several excellent review chapters within the recent book Solar Prominences (Engvold and Vial 2015) cover our theoretical understanding of the origins of such magnetic structures and their evolution on a variety of temporal and spatial scales (Mackay 2015), the energetic processes associated with prominence formation and visibility (Gilbert 2015; Heinzel 2015; Karpen 2015), and the mechanisms responsible for the ultimate fate of many prominences as the cores of solar eruptions (Fan 2015). Prominence observations

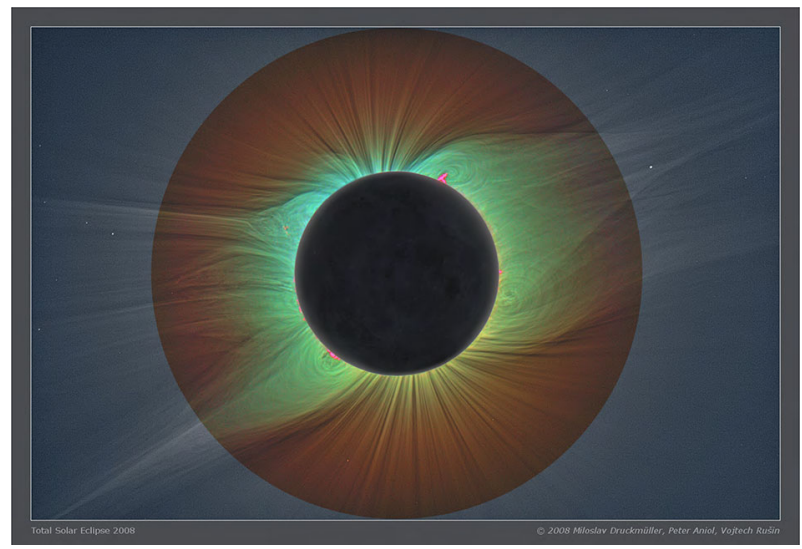

Fig. 1 Prominences in true color during 2008 total solar eclipse. Image reproduced with permission from http://www.zam.fme.vutbr.cz/ druck/eclipse/, copyright by Miloslav Druckmüller, Peter Aniol, Vojtech Rušin 
and interpretation are also well-covered by other chapters within that book (Ballester 2015; Engvold 2015; Gibson 2015; Gopalswamy 2015; Kucera 2015; Labrosse 2015; López Ariste 2015; Lugaz 2015; Martin 2015; Parenti 2015; Webb 2015) and by the Living Reviews on "Prominence Oscillations" by Arregui et al. (2018), and "Solar Prominences: Observations" by Parenti (2014). Additional comprehensive reviews of prominence structure, dynamics, and physical processes are found in Labrosse et al. (2010) and Mackay et al. (2010). Finally, for a big-picture context to prominence theory and models, we refer the reader to Low (2018), a recent review on "Coronal Magnetism".

The focus for this review is the physical state of the non-erupting prominence, i.e., the nature of its magnetohydrodynamic (MHD) quasi-equilibrium. In particular, in Sects. 2 and 3, we review models of the magnetic structure-or skeleton-of the prominence and its coronal environment. In Sect. 4, we consider prominence dynamics and thermodynamics, and present a brief overview of the state of the art in numerical simulations, where MHD is coupled to energy transport. Finally, in Sect. 5 we reflect upon some general conclusions and expectations for the future.

\section{The prominence magnetic skeleton}

Prominences are in many ways heterogeneous. They vary spatially in size and location, and vary temporally in evolution and dynamics. This complicates statistical analyses and classification schemes (Tang 1987; Mackay et al. 2008; Engvold 2015). Even so, many commonalities are observed (Parenti 2014). Over the past several decades, these have been used to build physical models of the prominence.

The generality and applicability of these models can be explored by comparing to a variety of observations, and the models themselves made more "realistic" through the use of complex magnetic boundaries. It is important to bear in mind, however, that as we will discuss in Sect. 4-prominence dynamics and thermodynamics challenge many of the assumptions of these relatively simple models. Nevertheless, such models provide a framework for understanding the prominence as a fundamental magnetic structure of the solar corona.

\subsection{Prominence bones: magnetic dips}

The central portions, or "spines", of prominences are generally observed to be localized to a vertical sheet suspended above and at an angle of about $20^{\circ}-35^{\circ}$ to an underlying magnetic polarity inversion line (PIL; Parenti 2014). Early observations of prominence magnetic fields found predominantly horizontal magnetic fields within this vertical sheet of a magnitude implying that magnetic pressure dominates over the thermal pressure in the prominence (low plasma $\beta$ ), and with this magnitude increasing with height (Rust 1967; Leroy 1977, 1978) (see Sect. 4.2.2 for further discussion). Since a low- $\beta$ plasma equilibrium is one in which magnetic forces balance ("forcefree" fields), these observations taken together imply a downwards magnetic pressure gradient force which in turn requires an upwards magnetic tension force. These char- 
acteristics, along with the observed longevity and general stability of prominences, led to a fundamental building block for early prominence models, namely, the requirement that the cool prominence mass be supported within dipped magnetic fields. (Note that we will revisit the absolute necessity for this requirement in Sect. 4.2.1.)

\subsection{Early prominence models}

One of the first two-and-a-half dimensional (2.5D) models proposed by Kippenhahn and Schlüter (1957) (KS) presented an idealized prominence as an infinitely thin and long vertical sheet of dense material standing on its edge above a solar PIL, supported in an arcade of dipped magnetic field lines oriented perpendicular to the PIL (Fig. 2a). Figure 2b, c represent a version of this model discussed by KS that is of particular interest because it is both analytic and stable. The arcade in this solution is quadrupolar, which enables dipped fields within an initially potential (current-free) magnetic field and so a stable environment for prominence formation (Fig. 2b). As the prominence mass accrues, the magnitude of the magnetic dip increases and a localized current sheet forms, producing an upwardly-directed Lorentz force that supports the prominence mass against gravity (Fig. 2c). Note that prominence mass is fundamental to the presence of currents in this particular model, in that the upward Lorentz force associated with the current sheet is directly balanced by gravitational forces acting on the prominence mass $\rho$ : i.e., $\rho * g=B_{x}(x=0) *\left[B_{z}\right]$, where $\left[B_{z}\right]$ is the jump across

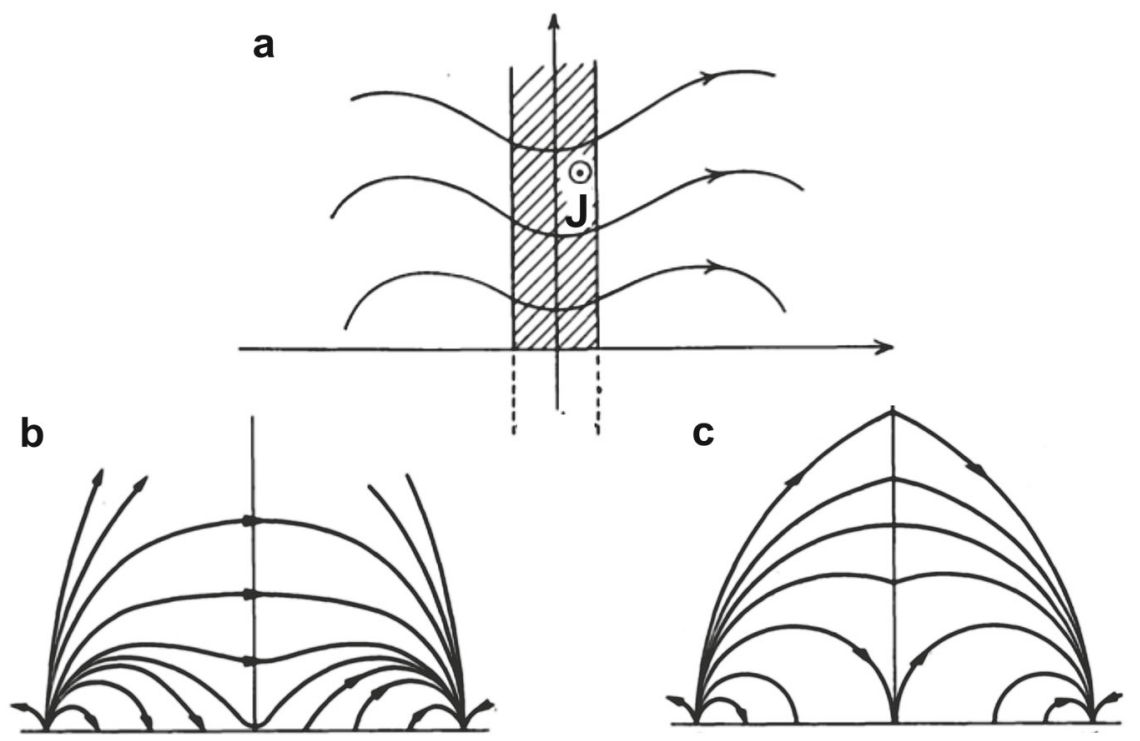

Fig. 2 Cross sections of 2.5D arcade-type prominence models. a Summary representation of Kippenhahn and Schlüter concept (note, current is pointing out of the page.) Image reproduced with permission from Anzer (1969), copyright by D. Reidel. b Example of analytic quadrupolar potential configuration with dips suitable for the formation of a stable prominence, that $\mathbf{c}$ may evolve to a non-potential configuration where prominence mass is supported by forces associated with a current sheet at the center of the dips. Image reproduced with permission from Kippenhahn and Schlüter (1957), copyright by Springer 


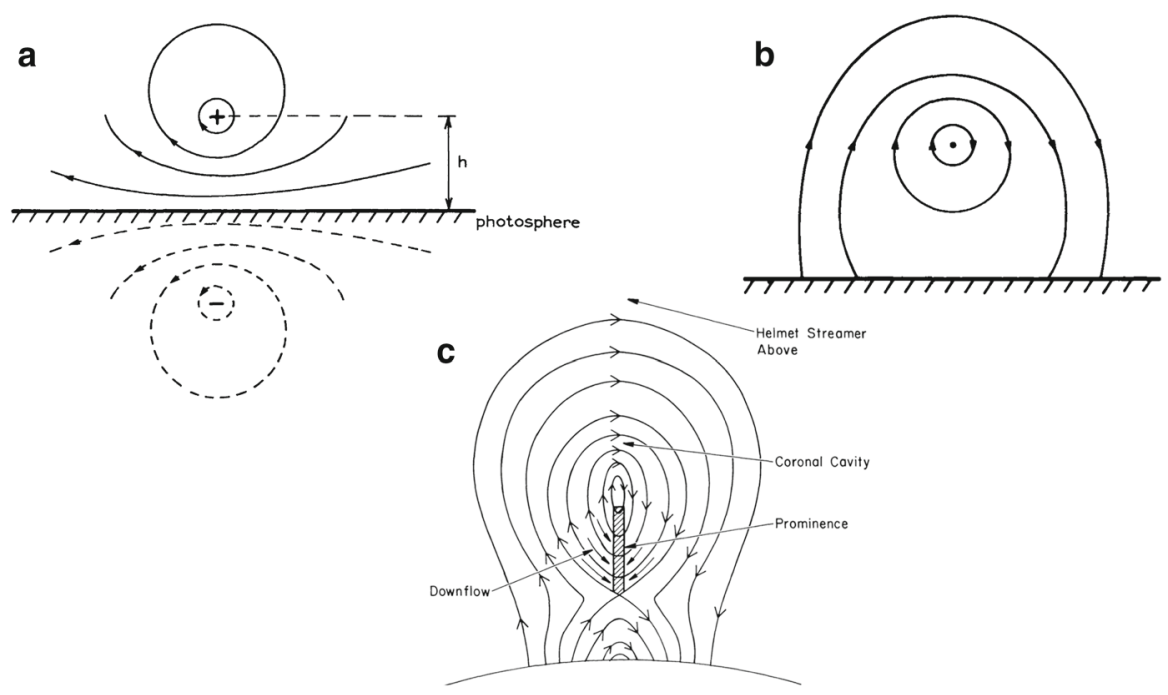

Fig. 3 Cross sections of 2.5D flux-rope type prominence models. a Kuperus and Raadu model field due to a current filament a distance $h$ above the photosphere (with image currents), and $\mathbf{b}$ the same embedded within a background potential field. Image reproduced with permission from Kuperus and Raadu (1974), copyright by ESO. c Summary configuration with the addition of X-line below prominence. Note that the current associated with the prominence axis is pointing into the page. Image reproduced with permission from Pneuman (1983), copyright by D. Reidel

the current sheet as $B_{z}$ switches sign (Anzer 1969). Thus, in the limit of prominence mass approaching zero, the current sheet vanishes and the configuration reverts to its initial potential dipped arcade configuration.

Another 2.5D model by Kuperus and Raadu (1974) (KR) considered the prominence to be aligned with a "current filament", detached from the photosphere and surrounded by a system of closed field lines (Fig. 3a). Induced currents at the photosphere repel those of the current filament, creating an upwardly-directed expansion force that provides support and stabilization against gravity for the prominence mass. A background field possessing horizontally-directed magnetic fields provides an additional confining downward force (e.g., Fig. 3b). In this scenario, the height at which the structure finds equilibrium can be affected by the mass of the prominence, and indeed, the authors argued that mass draining could lead to an increase in that height and potentially a loss of equilibrium (see also discussion in Low 1996). Since the prominence is magnetically-dominated, a component of the prominence magnetic field aligned parallel to the currents is needed to avoid destabilizing magnetic pinch effects. In the limit of prominence mass approaching zero, therefore, an equilibrium may exist in the form of a force-free magnetic flux rope containing field-aligned currents (Kuperus and Raadu 1974).

It is important to note that the prominence mass in the KR model may deepen the helical magnetic dips it accumulates in, creating a localized current sheet akin to that of the KS model with its own upwardly-directed Lorentz force. Thus, if we zoom in to the vicinity of the prominence itself, the models are actually quite similar: compare 
for example Fig. 2a to the central portion of Fig. 3c. The difference that does exist is one of reflection-in the KS model, the dipped magnetic field of the prominence runs left to right and the current is pointing out of the page, while the situation is the opposite for the KR model. As we discuss below, this difference must be considered in the context of the larger-scale magnetic equilibrium structures that encompass the prominence.

\subsection{Inverse versus normal magnetic fields}

A key paradigm in the historical development of prominence models was that of inverse vs. normal magnetic configurations (Leroy 1989). This nomenclature represents a concept where the direction of horizontal magnetic fields associated with the prominence dips is compared to the magnetic polarities at the photosphere below. If the field in the prominence dip is oriented so that it points from positive to negative photospheric polarity, as would be the case in a simple arcade, it is referred to as normal. If it points from negative to positive polarity, it is called inverse. Early observations noted that an inverse configuration was in fact prevalent in prominences (Leroy et al. 1984), with 75-90\% of prominences observed to have magnetic field oriented in a direction opposite to that expected from a potential field extrapolation (Démoulin 1998). Those oriented in the same direction, i.e., possessing a normal configuration, tended to be associated with stronger field active regions (Bommier et al. 1994).

Considering for now the bipolar versions of the KS and KR models shown in Figs. $2 \mathrm{a}$ and $3 \mathrm{c}$, we see that the former represents a normal configuration and the latter an inverse one. However, it is not generally true that dipped arcades always have normal configuration and flux ropes always have inverse configurations. As was demonstrated by Malherbe and Priest (1983) and several other authors (Amari and Aly 1989; Démoulin and Forbes 1992; Démoulin and Priest 1993; Low and Zhang 2004), inverse arcades and normal flux ropes are also possible, even in 2.5D (see Fig. 4). Moreover, equating the KS model with the normal configuration and the KR model with the inverse configuration (as has often been done in the literature) can lead to confusion. Note that the quadrupolar model shown in Fig. $2 \mathrm{c}$-from the original Kippenhahn and Schlüter (1957) paper-is identical to Fig. 4d-which was presented by Malherbe and Priest (1983) as an example of an inverse "KR" field.

But is that quadrupolar arcade model in fact an inverse field? An extrapolation of its photospheric field should arguably identify it as a normal prominence, since the direction of the dipped magnetic fields is the same as for the equivalent potential field (Fig. 2b). If instead the prominence fields are compared to a potential field extrapolation using only the bipolar field directly beneath the prominence, it would be characterized as inverse (Démoulin and Priest 1993). Although this latter approach is effectively that used in observational surveys of prominences (e.g., Leroy et al. 1984), realistic magnetic boundary conditions for prominence models may include curved or even multiple PILs. Thus, while inverse vs. normal is a fairly straightforward concept for 2.5D bipolar magnetic configurations, it becomes ambiguous for more complex fields. 

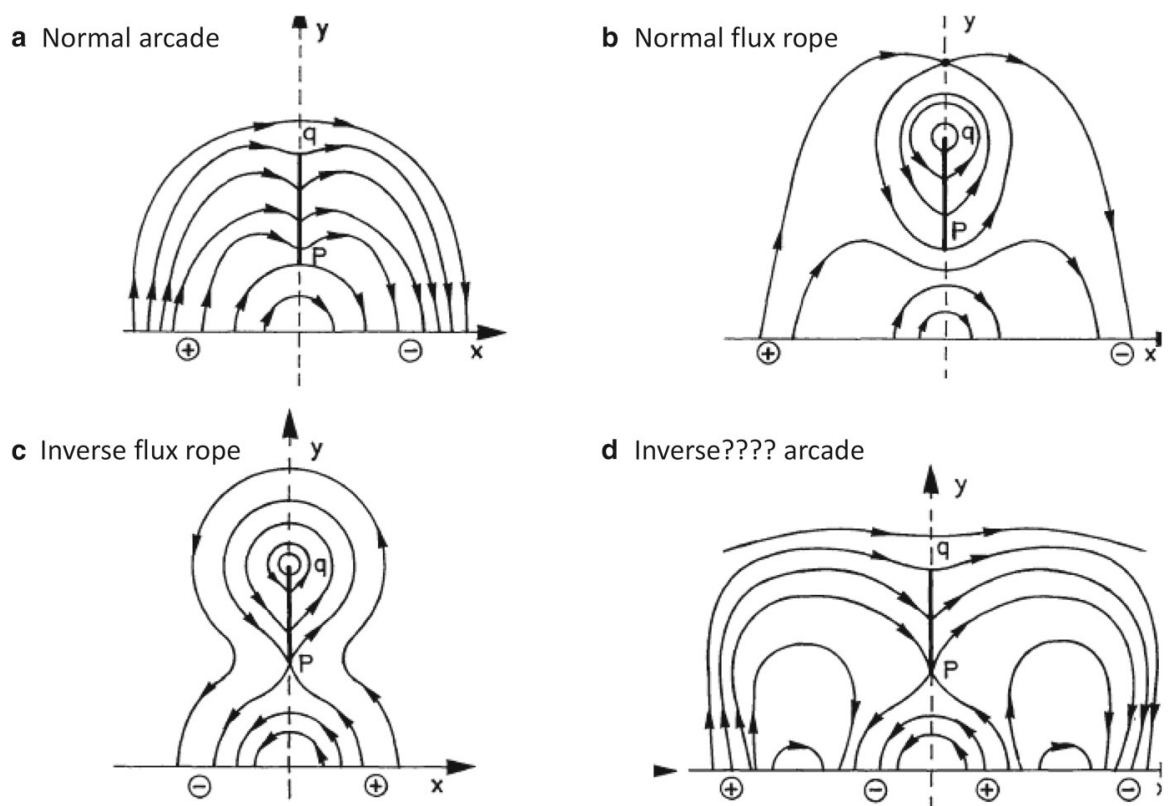

d Inverse???? arcade

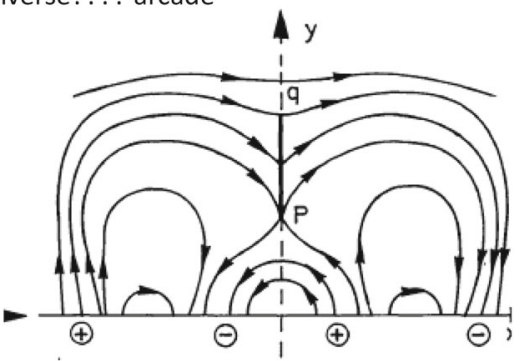

Fig. 4 Examples of inverse and normal magnetic configurations, for both dipped arcade and flux rope models. "????" in panel $\mathbf{d}$ indicates the ambiguity discussed in the text. Adapted from Malherbe and Priest (1983)

The dichotomy that is perhaps more meaningful, and that we now explore further in $3 \mathrm{D}$, is that of arcade vs. flux rope. A dipped (and in 3D, sheared) arcade is topologically equivalent to a potential arcade. This clearly distinguishes it - at least theoreticallyfrom a flux rope model, which possesses magnetic field lines that wrap around each other in a manner not obtainable to the potential field. As we will see, the challenge is to clearly distinguish these two topologies from each other observationally.

\subsection{Sheared-arcade versus flux-rope models}

\subsubsection{Sheared-arcade models}

The magnetic structure shown in Fig. 2c is a special case of a stable, dipped magnetic arcade with a current sheet that can be directly related to a dipped, fully potential field (Fig. 2b). It has the advantage of presenting a scenario in which the prominence may form in pre-existing dipped magnetic field that is the natural consequence of a particular photospheric magnetic flux distribution (Démoulin and Priest 1993). However, this distribution is quadrupolar, while the majority of prominences are associated with bipolar regions.

2.5D, bipolar, dipped-arcade models for prominences are possible, including configurations that are predominantly potential (Démoulin and Forbes 1992) or linearforce-free (Amari and Aly 1990; Démoulin et al. 1992) everywhere except for the 

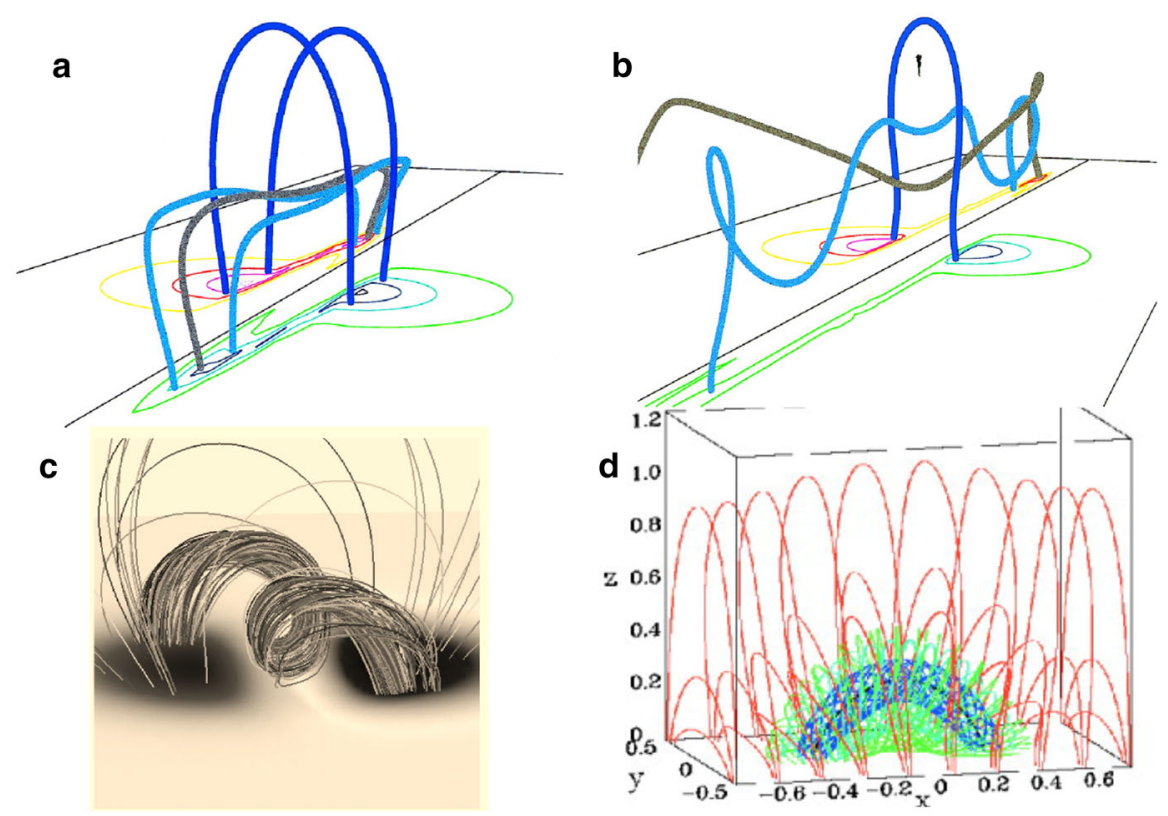

Fig. 5 3D magnetic configurations with dipped field capable of supporting prominences. Field lines from DeVore and Antiochos (2000) a sheared arcade and b flux rope; c Amari et al. (1999) and d Fan and Gibson (2004) flux ropes. Images reproduced with permission, copyright by AAS

prominence current sheet. However, such models beg the question of how the initial dipped configuration might arise. Since the critical element in a dipped bipolar arcade type topology is shear along the underlying neutral line, a logical approach would be to impose shearing motions on an arcade to create dipped fields. It is not possible, however, to form dips at the top of an arcade through shearing motions in $2.5 \mathrm{D}$, because field lines expand and become flatter as magnetic shear increases (Klimchuk 1990; Amari et al. 1991).

The case for a sheared arcade is much stronger in three dimensions, however, and (Antiochos et al. 1994) demonstrated how such a 3D force-free dipped arcade could be formed through shearing motions. In this case, the overlying arcade compresses the central part of the underlying sheared arcade locally, so that the dipped field is in the middle, rather than the top of the arcade (Fig. 5a). Moreover, the dipped fields in such a 3D sheared arcade are mostly inverse (Aulanier et al. 2002).

\subsubsection{Flux-rope models}

At this point we must pause and clarify our definition of a flux rope vs. sheared arcade. By a flux rope we mean anything in which magnetic fluxes wrap around a central axis (or axes, in the case of a spheromak). This leads to topologically-distinct magnetic sub-systems, such that e.g., quasi-separatrix layers (QSL_Priest and Démoulin 1995) form at steep gradients in field-line linkage, or bald-patch-separatrix surfaces (BPSS- 
Titov and Démoulin 1999; see also Fig. 9) manifest discontinuities between flux rope and neighboring arcade field lines. We will return to this topic in Sect. 3.2.

Mechanisms that have been proposed to create flux ropes include twisting motions at the photosphere (Priest et al. 1989), reconnection between sheared field lines (van Ballegooijen and Martens 1999), and emergence of pre-twisted fields from beneath the photosphere (Rust and Kumar 1994). Analytic flux-rope models evolved from 2.5 D, mostly force-free (Ridgway et al. 1991; Forbes and Isenberg 1991; Amari and Aly 1992; Low 1993; Schonfelder and Hood 1995) or magnetostatic configurations (Low and Hundhausen 1995), to 3D cylindrical (Titov and Démoulin 1999; Low and Berger 2003) and spheromak models (Lites and Low 1997; Gibson and Low 1998).

Further variety in 3D nonlinear force-free flux ropes has been enabled through numerical modeling. In particular, more realistic flux ropes embedded in overlying, near-potential arcades were formed through shearing followed by reconnection (DeVore and Antiochos 2000) (Fig. 5b), shearing followed by twisting motions or diffusive processes (Amari et al. 1999) (Fig. 5c), or emergence of twisted field into an overlying potential arcade (Fan and Gibson 2004) (Fig. 5d). Most of these models result in flux ropes that are not tightly wound, but rather possess between one and two turns about a central axis. Indeed, flux ropes that have more twist are prone to the kink instability, which may trigger eruption (Rust and Kumar 1996; Kliem et al. 2004; Török et al. 2004; Fan 2005). However, the kink instability may actually trigger a transition to a more tightly-wound topology, if such a topology represents a lower magnetic energy state. This has been demonstrated in a simulation where reconnections between an erupting, kink-unstable cylindrical flux rope and surrounding arcade fields resulted in a spheromak topology (Gibson and Fan 2008); arguably a quiescent spheromak field might arise in the case of a confined eruption (see, e.g., Török and Kliem 2005).

\subsubsection{Comparing to prominence properties}

So, can the basic observed properties of prominences (as described in Sect. 2.1) distinguish between magnetic topologies? We have presented models that possess significant three-dimensional magnetic complexity, but that so far do not consider the thermodynamics and radiative transfer problems necessary to fully model the cool prominence in the hot corona (we will return to this in Sect. 4). However, since we expect the prominence mass to be localized in dipped magnetic field (we will revisit this assumption in Sect. 4.2.1), we can extract a proxy for the location of prominence mass by "painting the dips" within any modeled magnetic field. The justification for this approach is that localized cross-field currents supporting prominence mass do so without substantially deforming the larger-scale force-free magnetic skeleton (we will revisit this assumption in Sect. 4.2.2). Because the pressure scale height for the relatively cool prominences is much smaller than for coronal plasma, the prominence may only fill a very small portion at the lowest portion of each magnetic dip (and, depending on the local thermal environment, may not fill even that). The result will be bead-like condensations that collectively form the prominence (see discussion in Low 1982; also Fig. 6a, b). 

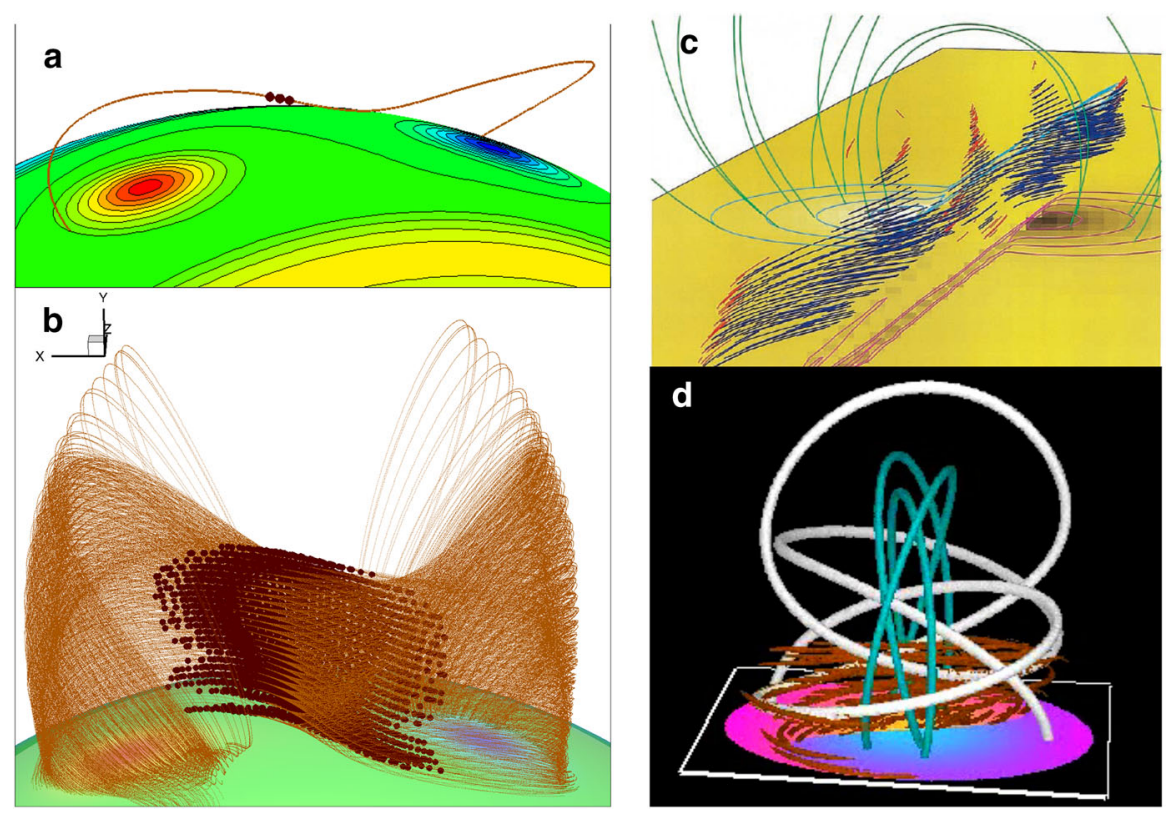

Fig. 6 "Painting the dips" of a magnetic skeleton results in sheet-like prominences, independent of magnetic topology. a Demonstration of the small region this would represent for a sample, dipped field line within a flux rope field model, and $\mathbf{b}$ demonstration for many field lines within the rope (after Gibson and Fan 2006a). c Painted dips within 3D sheared-arcade model. Image reproduced with permission from Aulanier et al. (2002), copyright by AAS. d Painted dips within 3D analytic model of spheromak-type magnetic flux rope. Image reproduced with permission from Lites and Low (1997), copyright by Springer

Figure $6 \mathrm{~b}, \mathrm{~d}$ show the loci of dipped magnetic field lines for models ranging from sheared arcade to cylindrical flux rope to spheromak flux rope (all of which have predominantly inverse configurations-see Sect. 2.3). From this we see that these disparate topologies all result in a sheet-like prominence structure even though there are no obvious sheet-like discontinuities in the surrounding magnetic skeleton. Counterintuitive though it may seem, even the highly twisted spheromak magnetic field results in a vertical sheet that makes only a slight angle to the underlying neutral line.

The models we have discussed so far have been idealized, with homogeneous, mainly bipolar photospheric boundaries. More realistic models would require solving a "mixed boundary problem" that takes into consideration both the prominence and the full complexity of the lower magnetic boundary. (See Démoulin et al. 1992 for a discussion of the historical development of the mixed boundary problem as applied to prominences.) Extrapolations from photospheric magnetic observations have explicitly incorporated a prominence in the form of an inserted flux rope in fitting linear (Aulanier et al. 1999) and nonlinear (van Ballegooijen 2004; Su and van Ballegooijen 2012) force-free models. In some cases, observations of coronal structures have also been used to constrain such magnetic extrapolations (Savcheva and van Ballegooijen 2009; Malanushenko et al. 2014). With this in mind, we now turn to other signatures of the magnetic structures that encompass prominences. 


\section{Beyond bones: the prominence in context}

In Sect. 2, we discussed how prominences (which we will also refer to as filaments when viewed against the solar disk) are observed to form above photospheric polarity inversion lines. Additional observed properties of their environment include the fact that they form within magnetically-defined filament channels, and that closed magnetic fields surround them, manifesting as an overlying helmet streamer, coronal cavity, and/or system of sheared, sometimes sigmoidal, coronal loops.

\subsection{Filament channels and chirality}

Filament channels are regions where chromospheric fibrils are aligned on either side of a polarity inversion line (Fig. 7a). They are arguably "more fundamental than filaments" (Mackay 2015), since a filament channel may exist without a filament, but a filament cannot exist without a filament channel. The patterns formed by the fibrils as they relate to surrounding magnetic fields has been interpreted as a indication of magnetic chirality, or handedness (Martin 1998a). In particular, when viewed from the positive polarity side, if the fibrils on the negative side of the filament channel extend to the right, they are referred to as "dextral", and if they extend leftward, they are referred to as "sinistral".

Filament barbs are also observed to extend from the central spine (Fig. 7b). The tendency of these to bear left or right (as viewed from the positive polarity side) is another measure of chirality that consistently matches that observed for the filament channel fibrils. These barbs are associated with small regions of opposite, or "parasitic" magnetic polarity (Martin 1998a). Aulanier and Démoulin (1998) built a linear forcefree flux rope with a periodic boundary to demonstrate that the introduction of parasitic polarities to a larger-scale bipolar photospheric flux distribution resulted in magnetic dips organized in a manner reminiscent of such barbs extending out from the central sheet (Fig. 8). However, there is some controversy regarding the magnetic structure of barbs, and how these relate to flows in prominences (Chae et al. 2005). We will return to the question of flows vs. field in Sect. 4.1).

Finally, the coronal loops observed above filaments have their own indication of chirality. In this case, however, the overlying structure skews at an angle that opposes the underlying filament and fibril structures. In particular, as Fig. 7c illustrates, a righthanded (dextral) filament channel always develops beneath a left-skewed coronal loop system, and vice versa (Martin and McAllister 1996).

Taken together, the observed chirality properties of filament channel, filament, and overlying arcades are easily reconciled with a magnetic flux rope topology; in particular, the overlying skewed arcade is a natural consequence of a helical system where the tops of winding field lines have the opposite orientation vs. the bottoms relative to the underlying PIL (Fig. 9a). Similarly, Gibson and Low (2000) described how a spheromak topology could also result in these chirality properties. However, a large amount of twist is not necessary and indeed simulations of what many would call sheared arcades (possessing field lines that wind less than a full turn about a central axis) also result in oppositely directed skew for overlying field lines vs. lower-lying, 


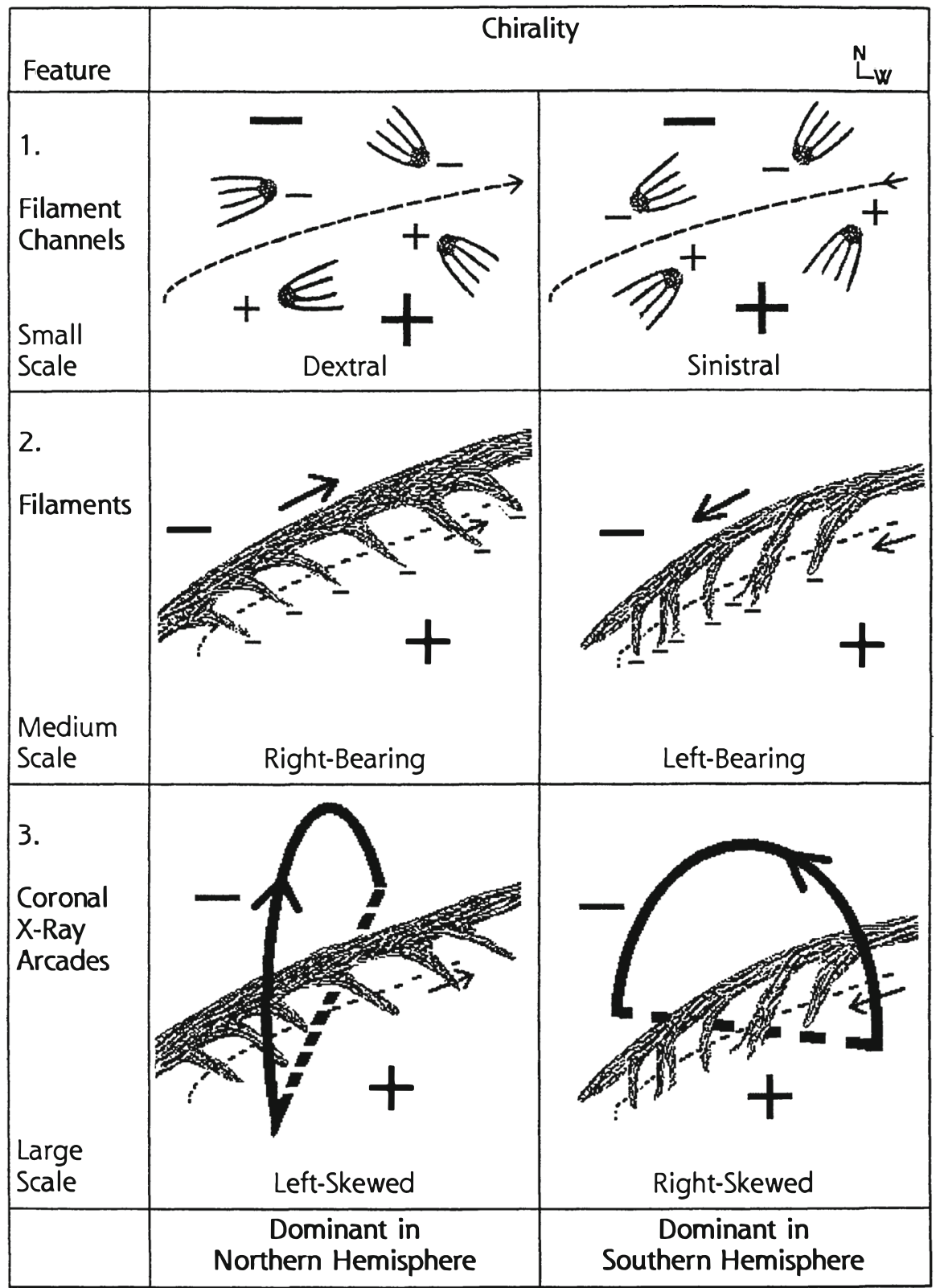

Fig. 7 Chirality relations between prominence(filament) and surrounding environment. 1 Chromospheric fibrils observed in filament channels; 2 filament extensions (barbs) from central spine; and $\mathbf{3}$ overlying coronal loops. Note this is the classic sketch from Martin (1998b), copyright by ASP-for an updated sketch see Fig. 7 of Martin (2015). This recent review emphasizes that proper chirality determinations require adequate spatial resolution and/or flow patterns to aid in seeing the direction of filament threads, as well as consideration of the perspective from which a filament is viewed 
a Sinistral

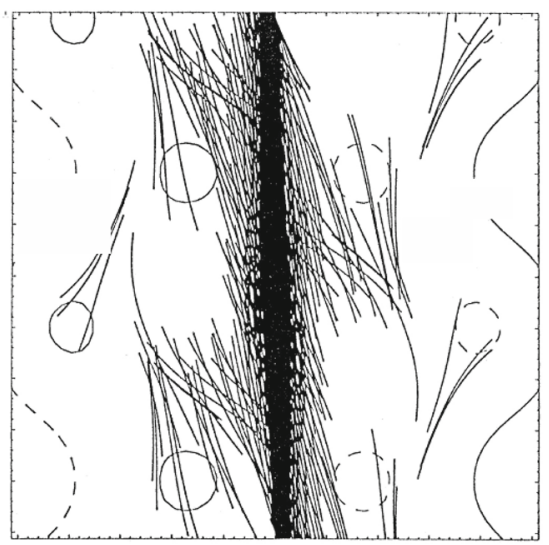

b Dextral

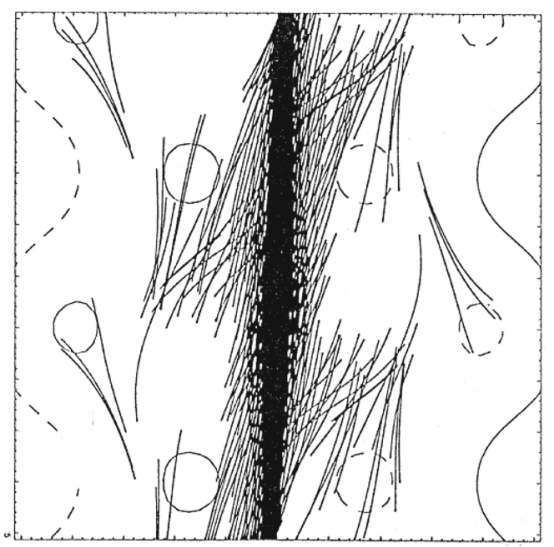

Fig. 8 Adding parasitic polarities to the photospheric flux distribution results in a linear-force-free flux rope with filament barbs. Dark lines represent dips in magnetic field lines consistent with a left-handed (sinistral) and b right-handed (dextral) chirality barbs. Image reproduced with permission from Aulanier and Démoulin (1998), copyright by ESO; see also Aulanier et al. (1998)
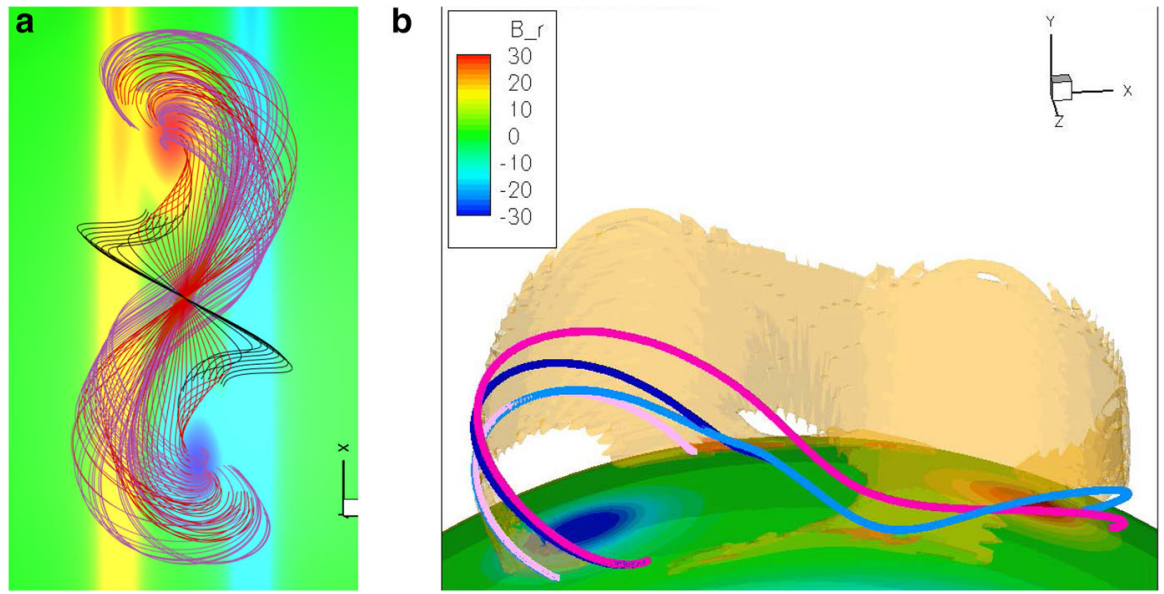

Fig. 9 Equilibrium magnetic flux rope model and sigmoidal separatrix. a Demonstration of the oppositely directed field-line orientations relative to the PIL manifested at the bottom versus top of a right-handed helical flux rope. Magenta field lines represent the bald-patch-separatrix surface (BPSS) and trace out the bottom of the helix as it grazes the photosphere, while black field lines represent the oppositely oriented field lines at the top of the flux rope (after Fan 2017). b Current sheet (isosurface) that forms under dynamic (but not eruptive) perturbation, leading to interchange reconnections between BPSS (light-blue) and adjacent arcade (dark-blue) field lines and resulting in reconnected sigmoidal/arcade field lines (dark pink/light pink). Image reproduced with permission from Gibson and Fan (2006a), copyright by AGU

dipped field lines (e.g., Welsch et al. 2005). For all of these models, the opposite orientations observed for filament vs. overlying arcade does not require opposite signs of magnetic helicity (see discussion in DeVore and Antiochos 2000). 


\subsection{Sigmoids and separatrices}

Soft-Xray (SXR) "sigmoids" are active regions with a characteristic S or inverse-S shape (Rust and Kumar 1996) (Fig. 10a). These regions are prone to eruption (Canfield et al. 1999; Glover et al. 2000), leading to the interpretation that the sigmoidal shape indicates non-potentiality, or energization. Sigmoids range from regions of sheared loops which collectively create an S- or inverse-S-shaped pattern, to sharply defined individual sigmoidal loops. The former may be long-lived, manifesting for multiple days, but the latter tend to be transient. Such transient sigmoid loops may exist stably for several hours before an eruption onset (Green and Kliem 2009; James et al. 2017). They also may appear and disappear without eruption over the course of several days for a given region (Gibson et al. 2002).
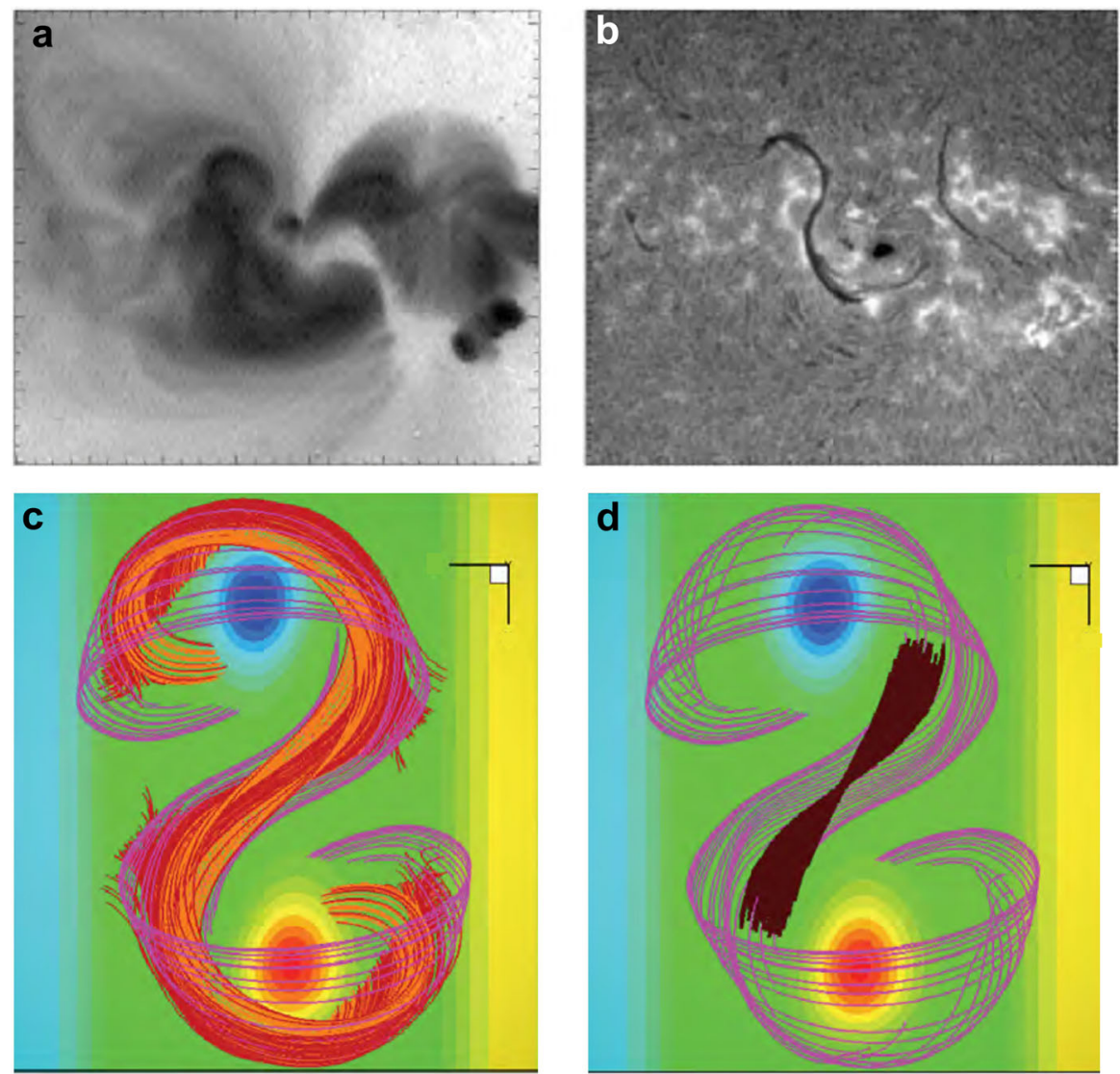

Fig. 10 A flux-rope model filament lies above and within the BPSS identified with a SXR sigmoid. a Yohkoh SXT (inverse color scale) and b Big Bear Solar Observatory (BBSO) observations of a SXR sigmoid and $\mathrm{H} \alpha$ sigmoidal filament. Image reproduced with permission from Gibson et al. (2002), copyright by AAS. c Flux rope with BPSS (purple) and current-sheet-intersecting field lines (red/orange). Orange field lines in c have dips, and $\mathbf{d}$ paints the centers of these and other dipped field lines up to a prominence scale height (brown). Image reproduced with permission from Gibson and Fan (2006a), copyright by AGU 
Filaments are often associated with SXR sigmoids, and may exhibit a sigmoidal shape as well (Aurass et al. 1999; Gibson and Low 2000; Pevtsov 2002; Gibson et al. 2002) (Fig. 10b). Unlike the overlying arcades observed by Martin and McAllister (1996), SXR sigmoids show the same direction of skew vs. the PIL as associated filaments, such that $S$ (inverse-S) shapes are associated with positive (negative) chirality (Pevtsov et al. 1997; Green et al. 2007). An examination of Fig. 9a) in comparison to Fig. 8 reveals that this implies, at least for the flux-rope model, that the SXR sigmoid must be formed from field lines tracing out the bottom of the helix, rather than the top.

Various models have been put forward to explain sigmoids that are consistent with this observed chirality relation, and we now briefly highlight some representative examples (see also Gibson et al. 2006a; Green et al. 2007 for further discussion of these and other models). A combination of magnetic flux emergence and diffusive evolution may explain the long-lived sigmoid in terms of a sheared arcade of "J"-type loops, which then may reconnect to form an erupting flux rope and associated transient sigmoid (van Ballegooijen and Mackay 2007; see also Amari et al. 2000; Moore et al. 2001; Kusano 2005). Alternatively, an eruption in which a pre-existing rope kinks downward could create sigmoidal current sheet enhancements of the right orientation (Kliem et al. 2004).

Not every sigmoidal loop erupts, however, and other models address how they may form in quiescent magnetic structures in association with separatrix layers. In particular, QSLs (the areas where magnetic connectivity changes abruptly, see Sect. 2.4.2) are expected to be locations where current sheets form and reconnections occur (Démoulin et al. 1996; Titov et al. 2002; Aulanier et al. 2005). The nature and location of QSLs for flux ropes depends upon the rope's topology. If the rope has an arcade beneath it (see Fig. 3d), the QSL will incorporate the line of magnetic X points that separate the rope from arcade, and be prone to current-sheet formation beneath the rope (Titov et al. 2003; Galsgaard et al. 2003). Simulations have demonstrated that magnetic field lines intersecting this sheet will form a sigmoid in the correct direction (Kliem et al. 2004; Török et al. 2004). Indeed, the QSL may evolve from 2 J-shapes to an S-shape, as tether cutting reconnections continue to add twist to the rope and drive its slow rise (Fan 2012) (see also Aulanier et al. 2010; Savcheva et al. 2012b, c).

For more low-lying flux ropes, the QSL takes the form of a sigmoidal BPSS (see Sect. 2.4.2) consisting of field lines whose dips just graze the photosphere-forming the so-called "bald patch" (Titov and Démoulin 1999; Low and Berger 2003). As Fig. 9b) illustrates, current sheets form at the BPSS leading to interchange reconnections between J-type arcade and S-type flux-rope field lines (Gibson et al. 2004; Gibson and Fan 2006a). The existence of BPSS-associated sigmoidal loops has been demonstrated observationally by Green and Kliem (2009), who analyzed the transition from J-shaped loops to a sharply defined sigmoidal loop, showing that it had 3 PIL crossings (as expected for a BPSS) and remained visible for several hours before erupting.

Moreover, in some cases a sigmoid will survive eruption, along with part of a filament (Pevtsov 2002; Green and Kliem 2009; Tripathi et al. 2009). This can be explained via the bifurcation of an erupting flux rope (Gilbert et al. 2000; Gibson and Fan 2006b) (Fig. 11). In this case, some portion of the sigmoid associated with 
a

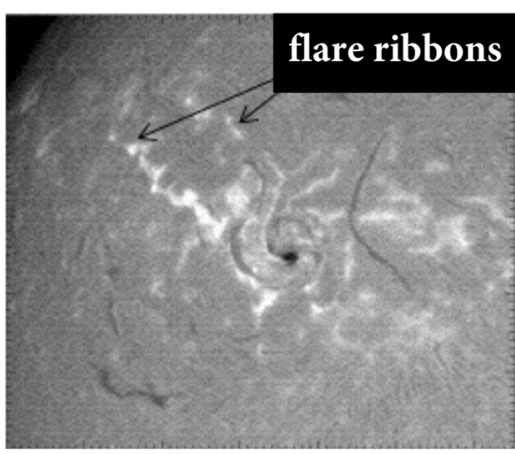

C

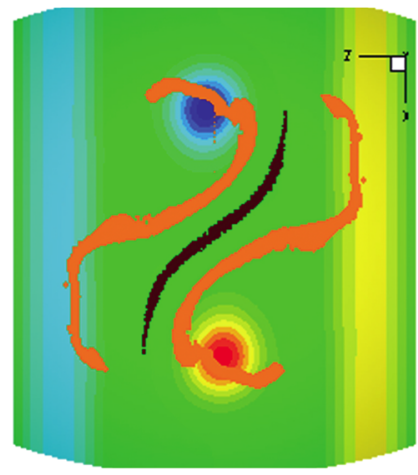

b

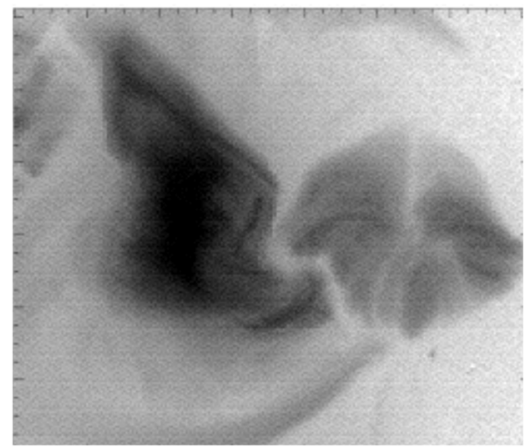

d

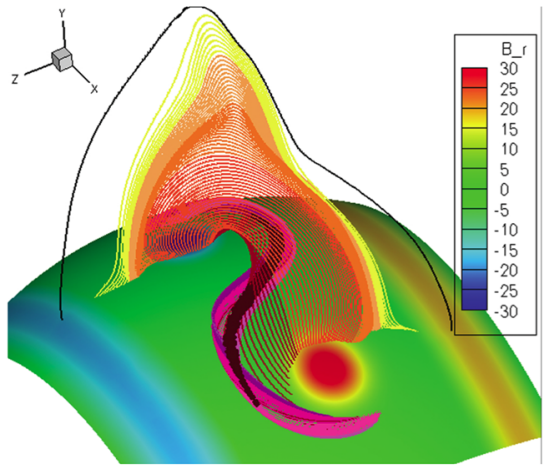

Fig. 11 Partially-erupting sigmoids/filaments. a BBSO $\mathrm{H} \alpha$ observations of flare ribbons surrounding a filament unaffected by the eruption and b Yohkoh SXT (inverse color scale) observations of a sigmoid with overlying cusped field lines. $\mathbf{c}$ Footpoints of reconnected field lines during partial flux rope eruption, and $\mathbf{d}$ field lines surviving the eruption, including cusped field lines (yellow/orange) overlying the remains of a BPSS (purple) and dipped field (brown). Note that the Yohkoh/BBSO observations of the eruption shown here are of the same region, but two days prior to the non-erupting sigmoid shown in Fig. 10. Images reproduced with permission from [top] Gibson et al. (2002), copyright by AAS, and [bottom] from Tripathi et al. (2009), copyright by ESO

the BPSS naturally survives the eruption because it lies below the filament (see, e.g., Fig. 10c, d), and Green and Kliem 2009 for further discussion.)

\subsection{Cavities and flux surfaces}

An examination of Fig. 6 demonstrates how_regardless of model—the prominence material is likely to fill only a fraction of the volume represented by its encompassing magnetic skeleton. When viewed along the prominence axis, this larger magnetic volume may become clearly detectable as a dark, elliptical cavity surrounding the prominence that extends along the line of sight (Engvold 1989; Tandberg-Hanssen 1995) (Fig. 12a). The cavity provides the silhouette of the "invisible man" (Gibson 2014) from which we can infer the full extent of the prominence magnetic skeleton. 


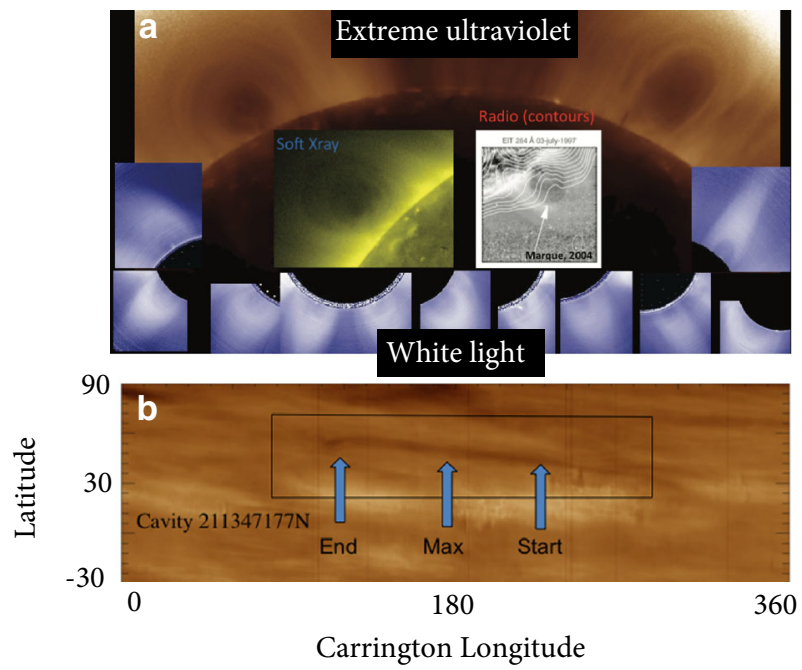

Fig. 12 Cavities are ubiquitous and three-dimensional. a Collage of cavities observed over a broad range of wavelengths. Extreme ultraviolet (EUV) observations from Solar Dynamics Observatory/Atmospheric Imaging Assembly (SDO/AIA); SXR from Hinode X-ray Telescope (XRT); white light images from Mauna Loa Solar Observatory Mk4 K-coronameter (MLSO/Mk4); Radio contours (Nancay) overlaid on Solar and Heliospheric Observatory EUV Imaging Telescope (SOHO/EIT) observations (Marqué 2004). Image reproduced with permission from Gibson (2014), copyright by IAU. b Carrington latitude versus longitude plot constructed from AIA coronal limb observations (cropped to emphasize northern latitudes), showing the presence of an tunnel-like cavity extended in longitude. Image reproduced with permission from Karna et al. (2015b), copyright by AAS

Cavity observations and models are reviewed comprehensively in Gibson (2015); we now briefly summarize the material presented there and in more recent publications.

Cavities are particularly evident for large, quiescent prominences-especially those that make up the polar crowns - and may last for days, weeks, or even months (Gibson et al. 2006b; Karna et al. 2017). Observations of such regions over multiple days and from different viewing angles have demonstrated that they are tunnel-like, sometimes with a croissant-shaped morphology (Gibson et al. 2010; Karna et al. 2015a, b) (Fig. 12b). They have been detected at multiple wavelengths, from radio to white light to EUV and SXR (Saito and Hyder 1968; Waldmeier 1970; Saito and TandbergHanssen 1973; Vaiana et al. 1973; Tandberg-Hanssen 1974; McIntosh et al. 1976; Straka et al. 1975; Kundu et al. 1978; Serio et al. 1978; Schmahl 1979) (Fig. 12a), indicating that they are regions of plasma depletion. However, their densities are still significantly higher than, for example, coronal holes; maximum cavity depletion is approximately 30-50\% relative to surrounding streamer densities (Marqué 2004; Gibson et al. 2006b; Fuller et al. 2008; Schmit and Gibson 2011). Cavities have coronal temperatures, but may be multithermal along the line of sight (Kucera et al. 2012), and may have a hot, central core (Hudson et al. 1999; Habbal et al. 2010; Reeves et al. 2012). This core may lie above the prominence and in the center of the cavity, like a lollypop on a stick (Fig. 13a). Horn-like brightenings may lie at the bottom interface of this core (Régnier et al. 2011); these have been demonstrated to emanate from the prominence and connect it to the cavity (Schmit and Gibson 2013). Finally, swirling 

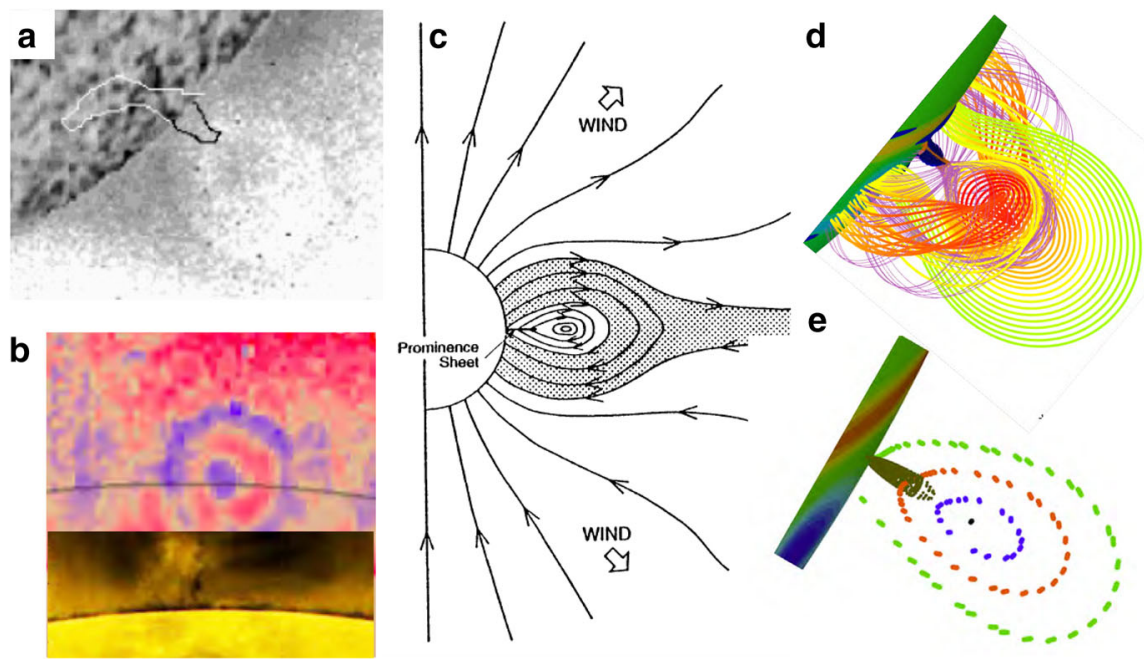

Fig. 13 Hot cores and bullseye flows exist within larger cavity volume, perched atop prominences. a Yohkoh observation of long-lived hot SXR core (inverse image); location of $\mathrm{H} \alpha$ prominence indicated by contour. Image reproduced with permission from Hudson et al. (1999), copyright by AAS. b (Top half) Bullseye lineof-sight flows observed by CoMP telescope within cavity; (bottom half) AIA 193 observations indicating presence of larger-scale cavity surrounding bullseye and central prominence (seen in absorption) beneath it. Image adapted from Bạk-Stęślicka et al. (2016). c Cartoon of cross section of flux rope embedded in closed and open fields. Image reproduced with permission from Low (1996), copyright by Springer. d Field lines within flux rope color-coded by temperature demonstrating the hot core lying above the dipped magnetic fields (brown) associated with prominence (from Gibson 2015); and e intersection of flux rope flux surfaces with plane of sky (colored dots). Image reproduced with permission from Forland et al. (2013), copyright by the authors

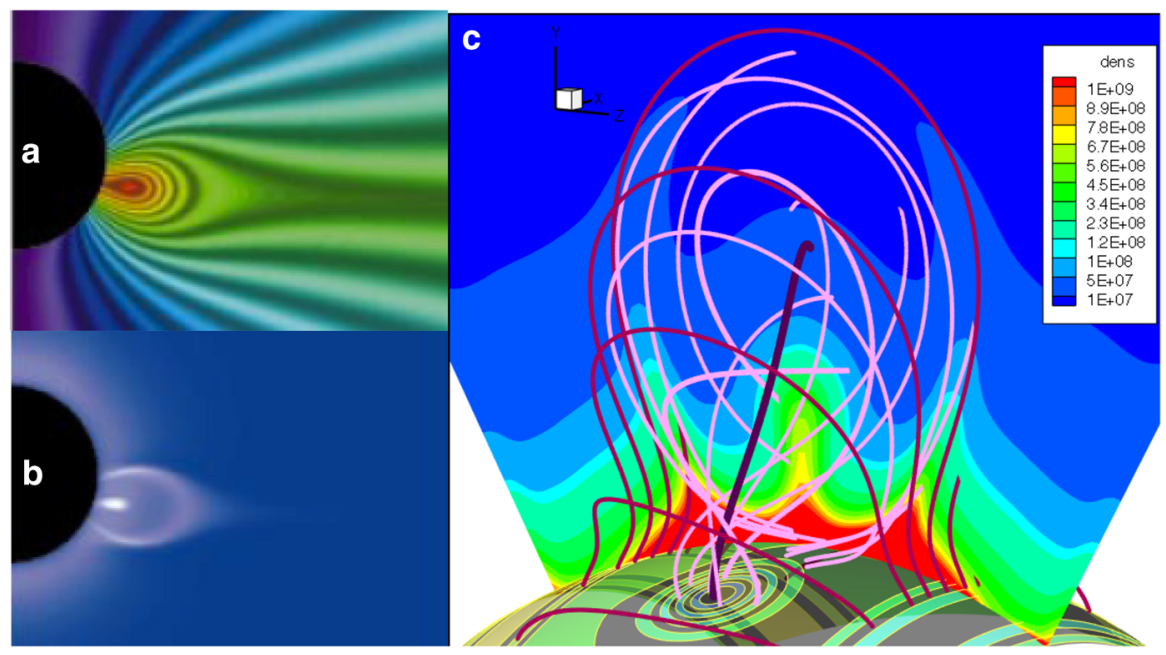

Fig. 14 Simulations in which cavities arise from pre-eruption magnetic flux ropes. a Magnetic field lines and $\mathbf{b}$ line-of-sight integrated polarized brightness simulation. Image reproduced with permission from Linker et al. (2003), copyright by AIP. c Field lines with central cut showing cavity in density (after Gibson and Fan 2006a) 

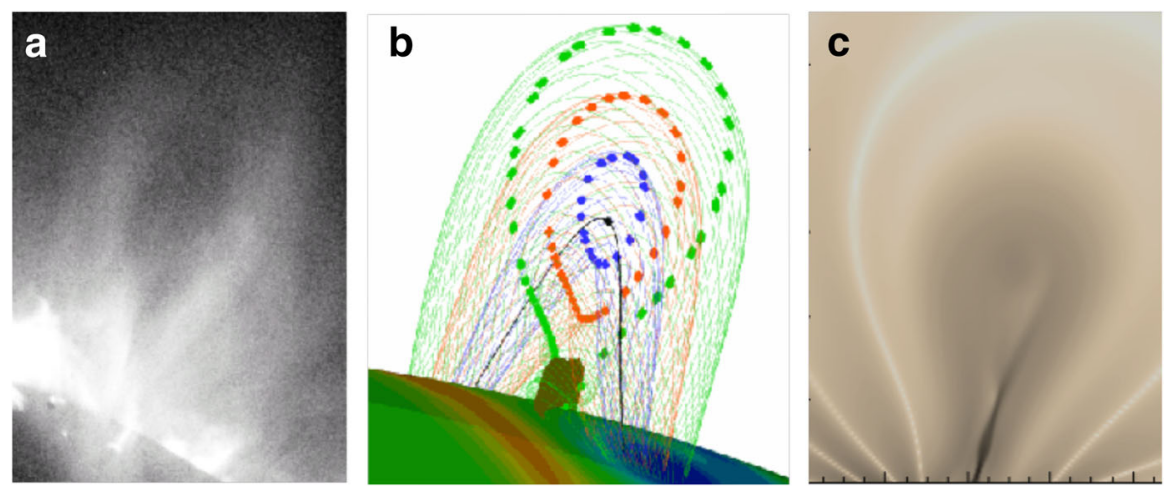

Fig. 15 Cavities with teardrop morphologies are likely to erupt. a Hinode XRT observations of a cavity $(01 / 11 / 2011$ 07:24UT) that erupted about $24 \mathrm{~h}$ later. Image courtesy K. Reeves. b Intersection of rope flux surfaces with plane of sky (colored dots). Image reproduced with permission from Forland et al. (2013), copyright by the authors - this may be compared to Fig. 13e which represents a flux rope with a BPSS, while the teardrop morphology of this figure represents a flux rope with an underlying X line. c Development of a teardrop morphology manifests as current enhancements (darker grey) within a torus-unstable erupting flux rope. Image reproduced with permission from Aulanier et al. (2010), copyright by AAS

motions have been observed within the cavity (Wang and Stenborg 2010; Li et al. 2012; Panesar et al. 2013), and line-of-sight flows of coronal plasma follow the contours of the cavity boundary (Schmit et al. 2009), in some cases forming a bullseye pattern within the cavity and above the prominence (Fig. 13b; Bạk-Stęślicka et al. 2016).

The idea that cavities indicate the presence of pre-eruption flux ropes has been argued for many years (Pneuman 1983; Low and Hundhausen 1995; Low 1994, 1996, 2001) (Figs. 3c; 13c) and demonstrated with analytic and numerical models in which cavities may exist in equilibrium before an eruption (Gibson and Low 1998; Linker et al. 2003; Fan and Gibson 2006) (Fig. 14; see also Sect. 4.3). The magnetic flux surface at the boundary between rope and arcade naturally gives rise to the sharp, elliptical boundary, and swirling flows and bullseye patterns are similarly explained by the nested toroidal flux surfaces of the rope's cross section (Fig. 13e). The relative locations of the prominence, hot central core, and surrounding large-scale cavity can all be explained in terms of the X-line/current sheet (Low and Hundhausen 1995), separatrix layers (Fan and Gibson 2006), and arched axial field lines within the rope (Schmit and Gibson 2014) (Fig. 13c, d). Explaining the density depletion in the cavity is somewhat more complicated; although force balance arguments can be invoked to require a strong axial magnetic field within the cavity to ensure total pressure continuity, this does not explain why the cavity is depleted in the first place (Low 1996). A variety of explanations are presented in Gibson (2015), including field-line length related thermodynamic effects and stability selection effects, and we refer the reader to that review for further discussion.

Cavities can bodily erupt as CMEs (Yurchyshyn 2002; Vršnak et al. 2004; Gibson et al. 2006b; Maričić et al. 2009), and, like prominences, may be activated prior to eruption exhibiting a slow rise, narrowing, and enhanced substructure. Even before activation, cavity morphologies provide clues to their stability. Forland et al. (2013) 
found that the majority of AIA cavities in a survey based on 2010-2011 observations were prolate (taller than they were wide). Karna et al. (2015a) had similar results. Both studies found that a prolate, and especially a teardrop-shaped morphology in cavities was a strong predictor of eruption (Fig. 15a). A theoretical explanation for this might be that teardrop-shaped flux surfaces occur when an X-line lies beneath a flux rope (Fig. 15b, c), effectively acting as a "lit fuse" in which tether-cutting reconnections at the associated QSLs lead to flux-rope axis rise and, inevitably, at least partial eruption of both cavity and prominence (Aulanier et al. 2010; Savcheva et al. 2012a, b; Fan 2015).

Because of the elliptical flux surfaces intrinsic to a magnetic flux-rope topology, and the evolution of these surfaces towards a tear-drop shape as the rope loses stability, the flux rope model is well-suited to explaining the observed properties of quiescent cavities that eventually erupt. However, although there are many observations of cavities existing stably prior to eruption (Koutchmy et al. 2004; Gibson et al. 2006b; Régnier et al. 2011; Forland et al. 2013; Gibson 2015), there are also cases where a cavity (bubble) apparently forms during the eruption, especially in association with impulsive eruptions from active regions (Patsourakos et al. 2010a,b). In such cases, a flux rope may form during the eruption, and the pre-eruption prominence may be better modeled by a sheared arcade. The question is whether the (relatively) small spatial scales and short temporal scales of active-region vs. quiescent prominences imply different enough physical environments to generally result in different topologies (e.g., sheared-arcade vs. flux-rope), or whether these spatial/temporal factors affect our ability to observe signatures of flux ropes. The signatures may also differ: rather than dark cavities, active region flux ropes may manifest as bright, hot features (Fan 2012, 2017). Such "hot flux ropes" may be associated with prominences and are generally seen in conjunction with eruptions; some apparently exist in advance of the actual eruption (Zhang et al. 2012; Patsourakos et al. 2013), while others apparently form during it (see Nindos et al. 2015 and references therein).

A final comment before we leave the subject of prominence cavities. Infrared polarimetric observations from the Coronal Multichannel Polarimeter (CoMP) (Tomczyk et al. 2008) consistently demonstrate a lagomorphic (rabbit-head-shaped) linear polarization structure associated with cavities. This polarimetric signature has been demonstrated to be consistent with that expected by a magnetic flux rope topology (Bâk-Stęślicka et al. 2013). These observations are not sufficient on their own to rule out the sheared-arcade model, however. Distinguishing between these topologies using coronal polarimetry requires larger-aperture solar telescopes capable of observing closer to the limb and with light-gathering capacity sufficient to obtain magnetically-sensitive circular polarization measurements (Rachmeler et al. 2013). Existing linear-polarization observations do, however, rule out a spheromak topology for most cavities (although CoMP observations consistent with a spheromak topology have been obtained at least for one cavity Dove et al. 2011). 


\section{Adding flesh and blood to the skeleton: incorporating dynamics and thermodynamics into prominence simulations}

The discussion until now has implicitly assumed some qualities of our magnetic skeleton that we will now call into question: that it is unchanging (at least when eruption is not imminent) and that it must be consistent with a force-free equilibrium plasma. We are driven to question these assumptions by an ever-increasing complexity of observations which directly or indirectly challenge them, and also by a commensurate evolution in the sophistication of prominence simulations.

\subsection{Prominence dynamics}

Prominences - even in their quiescent (non-eruptive) state-are intrinsically dynamic. The magnitude of these flows range from a few $\mathrm{km} / \mathrm{s}$ to a few $10 \mathrm{~s}$ of km/s (Parenti 2014). These flows are generally sub-sonic, and even more sub-Alfvenic-magnetosonic velocities in the lower corona are on the order of hundreds of $\mathrm{km} / \mathrm{s}$ (Warmuth and Mann 2005). Such flows do not themselves challenge the assumption of force-freeness (which we will return to in Sect. 4.2.2). However, as we now discuss, some prominence motions appear to violate the assumption underlying our entire concept of a magnetic skeleton: that is, that the plasma is "frozen" into the magnetic field and so all flows must follow its local direction. This is an assumption which holds whether or not the plasma is force free, and is a property of highly-conductive plasmas.

Field-aligned flows do appear to be common. We have discussed swirling coronal plasma within cavities; cool prominence material is also observed to flow, streaming in both directions along the prominence spine (Schmieder et al. 1991; Zirker et al. 1998), or moving apparently helically about the apparently vertical prominence barbs in "solar tornados" (Pettit 1932; Orozco Suárez et al. 2012; Wedemeyer et al. 2013; Su et al. 2014). Caution must be exercised, however, since projection effects or oscillatory motions may lead to false indications of rotational flows in tornados (Panasenco et al. 2014; Martínez González et al. 2016; Levens et al. 2016; Schmieder et al. 2017). There is also some ambiguity about whether prominence flows are along stationary magnetic field lines, or whether they represent motions of the magnetic structure itself (thus, a dynamic skeleton). Evidence for the latter was presented by Williams et al. (2009), who observed a whole prominence rotating about its spine, and by Okamoto et al. (2016), who observed a small part of a prominence spine rotating and interpreted it as magnetic reconnection between flux systems (Fig. 16).

The deeper puzzle lies in the fact that although observations of prominence magnetic fields indicate they are near horizontal (Leroy 1988), vertical flows, both up and down, are observed in prominence barbs and within vertical striated "hedgerow" prominences (see Berger et al. 2008 and references therein). Observations of $\mathrm{H} \alpha$ Doppler shift from the Meudon observatory indicated that the velocity vector is not aligned with the apparent vertical flows observed in intensity, but rather have a substantial horizontal component, consistent with flows tied to largely horizontal magnetic fields (Schmieder et al. 2010). This then begs the question of the cause of the apparent vertical structures and flows observed in intensity. 
a
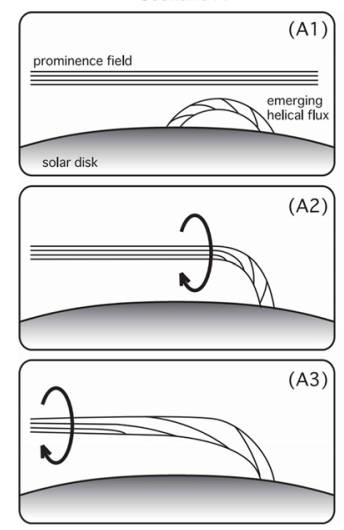

Scenario B

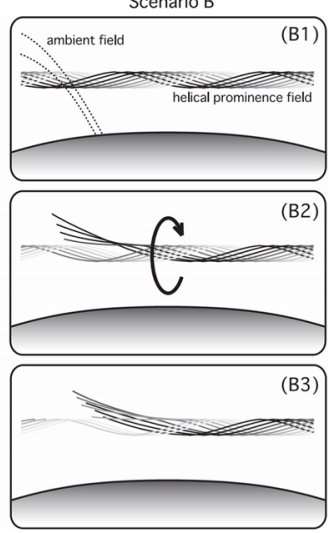

b
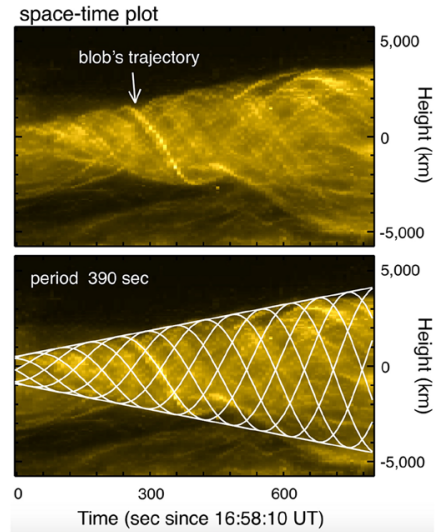

Fig. 16 Dynamic evolution of the magnetic skeleton may manifest as prominence motions. a Schematic representations of how reconnections between the prominence magnetic flux system and emerging field (Scenario A) or neighboring, ambient field (Scenario B) might result in reconnection and the transfer of helicity and magnetic flux between the systems, with associated flows. b Observations of fine structures in a prominence spine in the $\mathrm{Ca}$ II $\mathrm{H}$ line by Hinode/SOT are used to track the sinusoidal trajectory of a blob, which the authors interpret as evidence for Scenario B. Image reproduced with permission from Okamoto et al. (2016), copyright by AAS

a
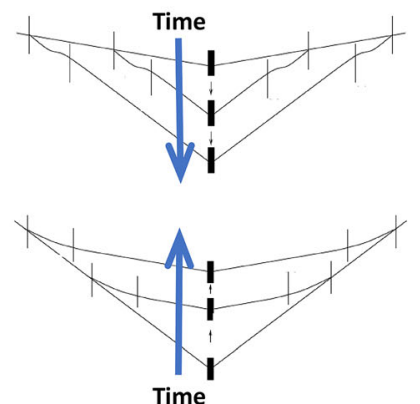

C

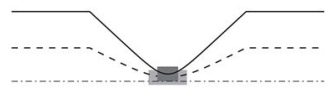

Shortly before reconnection

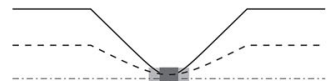

Shortly after reconnection

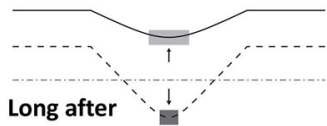

b

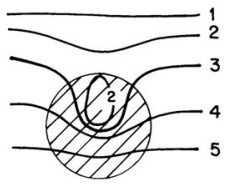

Time 0

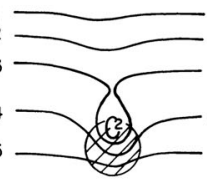

Time 1

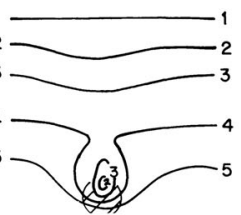

Time 2

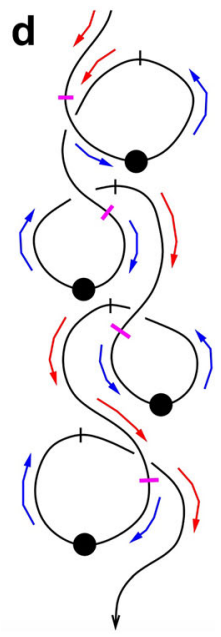

Fig. 17 Vertical flows in prominence sheets are possible even when the local magnetic field orientation is horizontal. a Constant-speed descent (top) or ascent (bottom) may arise in KS-type prominence sheets that are in local equilibrium but out of equilibrium with their supporting magnetic structure. From Low and Petrie (2005). b Gravity acting on prominence plasma may lead to sagging field lines that reconnect to form a descending knot of closed field. From Lerche and Low (1980). c Reconnections at tangential discontinuities may pass a parcel of prominence mass downward across neighboring field lines whose dips collectively form a vertical structure. From Chae (2010); see also Petrie and Low (2005). d Tangled magnetic field lines may possess sufficient horizontal magnetic field to support prominence mass against free fall. From van Ballegooijen and Cranmer (2010). Images reproduced with permission, a, c, d copyright by AAS; b copyright by Springer 

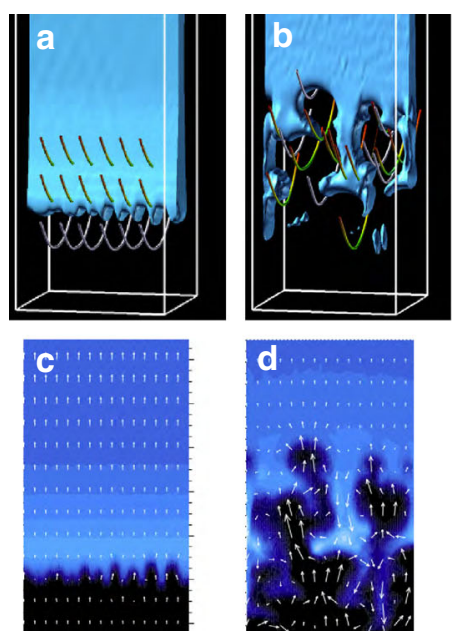

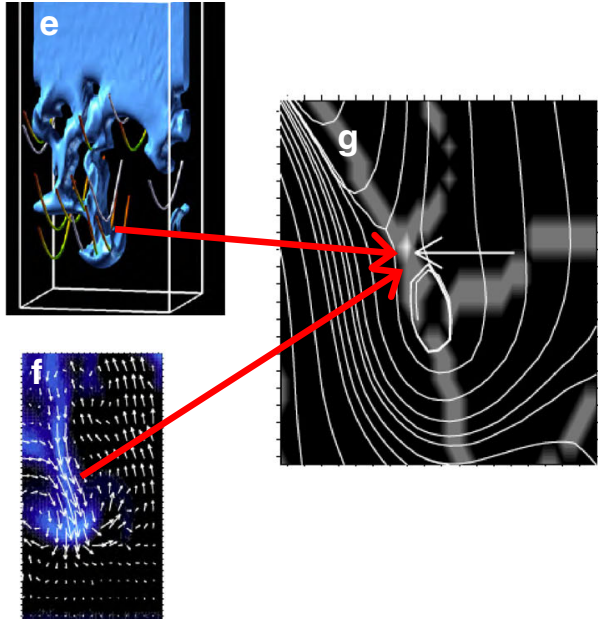

Fig. 18 Vertical flows in prominence sheets associated with the Magnetic Rayleigh-Taylor (MRT) instability. a A KS-type prominence model is perturbed by c a low-density/high-temperature region at its base, resulting in b/d RT unstable upward flows (from Hillier et al. 2012a). e-g Reconnections in this evolving plasma result in downward acceleration of fast-moving, dense blobs (from Hillier et al. 2012b). Images reproduced with permission, copyright by AAS

Vertically-aligned structures have been modeled as the superposition of dips in horizontal field lines (Low 1982; Heinzel and Anzer 2001) (see also discussion in Parenti 2014 and references therein). Vertical flows in such structures-both upward or downward - may arise due to local response to an imbalance between magnetic tension and gravitational forces (Fig. 17a), or from reconnection within and/or between sagging, dipped field lines (Fig. 17b, c) (Lerche and Low 1980; Petrie and Low 2005; Chae 2010; Low et al. 2012). Alternatively, van Ballegooijen and Cranmer (2010) modeled the prominences as a vertical sheet of tangled magnetic fields in which prominence mass moves downward via a series of subsonic and supersonic flows separated by shocks and sonic points (Fig. 17d). All of these models are consistent with locally horizontal magnetic fields in the prominence, and downward velocities much less than expected for free fall, as observed.

The downward draining of prominence material has been argued to represent the first half of a "magnetothermal convection" cycle, where the second half involves the replenishment of prominence mass through upward plume-like flows from apparently hot, tenuous bubbles of plasma, observed to form beneath some quiescent prominences (Berger et al. 2011). These upflows have been argued to indicate a magnetic RayleighTaylor (RT) instability between the coronal bubble and overlying prominence (Berger et al. 2010; Ryutova et al. 2010). The RT instability arises in general when heavy fluid lies above lighter fluid; such a situation can occur in a magnetized plasma, where, although total pressure (magnetic plus thermal) is required to be continuous across flux boundaries, thermal pressure is not. Magnetic fields actually play a stabilizing role, but only to perturbations parallel to the field (undular modes), and most strongly to short wavelengths. Perturbations perpendicular to the field result in the interchange of magnetic field lines. This has been demonstrated in a numerical simulation where a 
nonlinear perturbation to a KS dipped field model (see Sect. 2.2) resulted in upflows as field lines glide past each other (Hillier et al. 2011, 2012a) (Fig. 18a-d). Current sheets forming in the nonlinear phase of the RT instability then may lead to reconnections and downflows, completing the cycle (Fig. 18e-g).

Dudík et al. (2012) presented an alternative explanation for the plume upflows. These authors questioned the interpretation of bubbles and plumes as hot, arguing that they might rather be gaps in the prominence structure through which the background coronal emission became apparent. They demonstrated how a bubble consisting of arcade-type field lines might form below the dipped field of a linear-force-free flux rope perturbed by parasitic bipoles. They conjectured that the plumes were driven by reconnection at the magnetic separator outlining the bubble (see also Gunár et al. 2014). Further discussion of models of vertical flows in prominences, including a thorough review of prominence RT instability studies, can be found in Hillier (2018).

In summary-we cannot assume that our magnetic skeleton is static, or that flow lines necessarily indicate field lines. Prominence flows could also indicate a solidbody motion of the skeleton itself (e.g., Fig. 16), a breaking of it due to reconnection or deformation of it by gravity (e.g., Fig. 17), or an instability driving the rearrangement of magnetic field lines within it (e.g., Fig. 18). The skeleton itself might even be "leaky", with the diffusion of neutral atoms playing a role in prominence mass loss and dynamical instabilities (Mercier and Heyvaerts 1977; Gilbert et al. 2002; Khomenko et al. 2014), and/or the frozen-in condition spontaneously and recurringly breaking down (Low et al. 2012; Low and Egan 2014). This complicates the interpretation of observations, and motivates the development of comprehensive 3D numerical MHD simulations that explicitly take into consideration the dynamics - and as we now discuss, the thermodynamics — of the prominence system.

\subsection{Prominence thermodynamics}

At the risk of mixing metaphors: What makes a lake or a river? Topography clearly plays an essential role, but so does climate. Without frequent precipitation we are left with a dry river bed, or an empty lake. Similarly, a magnetic structure with a locus of dipped field lines may be conducive to supporting a prominence in equilibrium, but ultimately thermodynamics determines whether one actually forms.

But does this formation actually require an equilibrium? And, just as water may change the topography of the river bed it flows through, might the prominence plasma deform the topography of the magnetic field? It is clear that the $3 \mathrm{D}$ magnetic field that supports the prominence and the thermodynamic environment that enables its formation must be considered together if we are to answer these questions.

\subsubsection{Beyond equilibrium}

One dynamic approach developed to model prominence formation explicitly depends upon thermal nonequilibrium (TNE) (Antiochos and Klimchuk 1991; Antiochos et al. 1999, 2000). Here, heating at field-line footponts results in catastrophic cooling and condensation and forms a prominence that never reaches a static equilibrium, but 


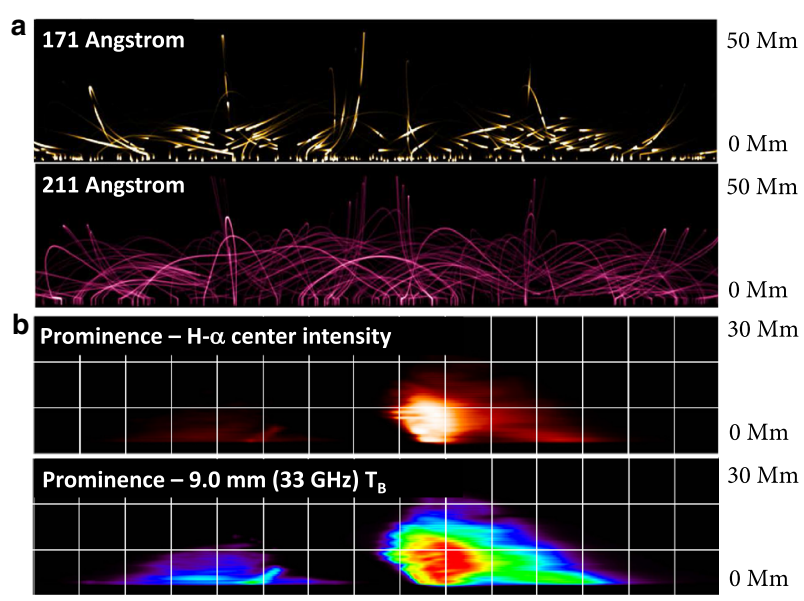

Fig. 19 Fine structure associated with prominences can be modeled within a 3D magnetic structure. a Snapshots of time-varying coronal emission at (top) $171 \AA$ and (bottom) $211 \AA$, for a 1D multithread simulation of TNE in a 3D sheared arcade (from Luna et al. 2012). b Prominence fine structure in (top) $\mathrm{H} \alpha$ emission and (bottom) $9.0 \mathrm{~mm}(33 \mathrm{GHz})$ brightness temperature determined from plasma in 1D hydrostatic equilibrium along field lines within a 3D NLFF magnetic model (from Gunár et al. 2016). Images reproduced with permission, copyright by AAS

rather is the product of an ongoing cycle of formation, motion, and destruction. Such a simulation raises the question: do we even need dipped magnetic fields for the prominence to form? To quote the pithy Karpen et al. (2001) abstract, "No." Long, flat field lines are sufficient for a steady-state solution. For further discussion of this and other prominence formation models, we refer the reader to the review by Karpen (2015), and references therein.

In modeling the full prominence magnetic skeleton, thermodynamic effects may be introduced by applying 1D simulations of plasma evolution to sampled magnetic field lines within a 3D magnetic model. In this way Lionello et al. (2002) demonstrated the condensation of cool material in magnetic dips of a 3D magnetic flux rope, solving steady-state hydrodynamic equations along field lines with conduction, heating, and radiative losses included. Luna et al. (2012) used TNE to investigate the fine-structure dynamics of the prominence and associated coronal emission, simultaneously solving TNE upon multiple field lines within a sheared arcade (Fig. 19a). Similarly, Schmit et al. (2013) applied TNE to sample field lines in a 3D flux rope model, providing clues to the prominence-cavity connection.

Although it did not explicitly model prominence formation, the Whole-Prominence Fine Structure (WPFS) model (Gunár and Mackay 2015a) also established a plasma distribution within a 3D non-linear force free (NLFF) magnetic field model (Mackay and van Ballegooijen 2009). This was conceptually similar to "painting the dips" as in Fig. 6, but done in a physically self-consistent way by imposing a semi-empirical temperature structure including a prominence-corona transition region and solving for hydrostatic balance (Gunár et al. 2013). This then allowed the generation of synthetic observations of, e.g., hydrogen emission through $1 \mathrm{D}$ radiative transfer calculations 
along multiple lines of sight (Heinzel et al. 2015), as well as brightness temperature and optical thickness at submillimeter wavelengths (Fig. 19b; Gunár et al. 2016). This is an intrinsically static approach; however, the time evolution of fine structures was also obtained in the form of a sequence of quasistatic equilibria by allowing the photospheric magnetic boundary to change, and with it the 3D NLFF magnetic skeleton (Gunár and Mackay 2015b). Thus, the WPFS approach obtained dynamics by evolving the magnetic skeleton itself, while the TNE approach was based on an unchanging magnetic skeleton, with flows due entirely to thermodynamics.

\subsubsection{Beyond force-free}

In Sect. 4.1, we discussed prominence dynamics that appear inconsistent with either thermally-driven flows along field lines or boundary-driven evolution of the skeleton itself, and discussed the possibility that some of these were due directly or indirectly to gravitational forces. This would violate the force-free assumption commonly made for coronal physics - that is, that magnetic forces self-balance and that ther$\mathrm{mal} /$ gravitational forces are tiny in comparison.

As discussed in Sect. 2.1, observations of prominence plasma and magnetic fields have motivated this assumption for decades: early calculations found plasma $\beta$, the ratio of thermal to magnetic pressures, of order $\approx 0.02$, based on average magnetic field measurements of $\approx 10$ Gauss and pressure of $\approx 0.1$ dyne $\mathrm{cm}^{-2}$ (Rust 1967). This study found field strengths of $\approx 5-10$ Gauss for most quiescent prominences, and higher strengths (60 Gauss) in active-region filaments, although the median values for active-region filaments were also in the 5-10 Gauss range. More recent observations have found patches of substantially higher magnetic field strengths (60-80 Gauss) in quiescent prominences (Casini et al. 2003), and even higher magnetic field strengths, on the order hundreds of Gauss, in active-region filaments (Kuckein et al. 2009). Luna et al. (2018) surveyed nearly 200 filaments near solar maximum, including both active region and quiescent filaments, and used prominence seisomology to establish minimum magnetic field strengths ranging from 2-38 Gauss, with an average of 17 Gauss. This study also found that the minimum magnetic field strengths did not differ significantly for quiescent vs. active-region filaments. All of these observations support the concept of generally force-free prominences.

It is clear, however, that magnetic field strengths are not homogeneous across the prominence, and similarly that their masses may vary significantly. Assuming magnetic field strengths of a few Gauss and modeling a massive, well-developed prominence, Anzer and Heinzel (2007) found plasma $\beta$ could be of order unity or higher in quiescent prominences. Such considerations, combined with the aforementioned prominence dynamics observations, motivate us to to consider the implications and interpretation of non-force-freeness in prominence simulations.

Hillier and van Ballegooijen (2013) examined the effects of mass loading an initially force-free, 2.5D flux rope, and relaxing it to a new MHD equilibrium. They found that increasing mass and/or plasma $\beta$ led to deformation of the magnetic field lines, in particular pulling the flux rope axis down. They argued that the most prominence-like distribution of mass (sheet-like and high density) occurred for cases where gravity was balanced by the additional magnetic tension force introduced by the deformation, and 
where $\beta$ (and with it, the thermal pressure gradient) possessed moderate values $(\approx$ 0.1 ). They thus concluded that magnetic forces were fundamental to the prominence support; however, thermal and gravitational forces could not be considered a small perturbation to the system.

Terradas et al. (2015) undertook a similar study using a 3D sheared arcade and found that the global morphology of the modeled prominence depended on plasma $\beta$, with lower $\beta$ plasma supporting detached prominences and higher $\beta$ resulting in mass extending down to the photosphere, in a manner reminiscent of hedgerow prominences. For low $\beta$ cases, they found evidence of Magnetic Rayleigh-Taylor (MRT) dynamic behavior. Terradas et al. (2016) then studied the dynamic evolution of mass inserted into a 3D force-free flux rope (Titov and Démoulin 1999). Here again the magnetic structure was pushed downward by the mass and the field lines slightly deformed, with the dips associated with flux-rope twist providing support against gravity in a low- $\beta$ regime. Interestingly, the MRT instability was suppressed and the prominence was structured horizontally along the rope axis, rather than vertically, due to the Kelvin-Helmholtz shear instability. The authors concluded from these two studies that horizontally-structure prominences may be more consistent with a fluxrope topology, while vertically-structured prominences may be more consistent with a sheared arcade.

In summary-we cannot assume that the prominence is in equilibrium, or that it is fully force-free. Prominence formation and evolution respond to an ever-changing thermodynamic and magnetic environment, and the environment in turn may be affected by the prominence plasma.

\subsection{The Full Monty: prominence formation in 3D MHD}

Finally, we turn to two recent MHD simulations that provide self-consistent representations of the prominence forming within a 3D magnetic skeleton. Both are flux rope models, and both explicitly treat the energy equation including conduction, radiative cooling, and coronal heating.

Xia and Keppens (2016) obtained their prominence in three steps. First, they constructed an isothermal flux rope in MHD equilibrium. Second, they added a hydrostatic vertically-structured atmosphere including a chromosphere, and solved the MHD equations with explicit treatment of the energy equation. Finally, they applied localized heating at the flux-rope foot points and condensed a prominence via TNE (Fig. 20a). The resulting prominence was fragmented and highly dynamic, with blobs and threads continuously forming in the dips of the flux rope and dragging the field lines downward to create vertical hedgerow-type structures. Prominence plasma was replenished through ongoing evaporation from the chromosphere and condensation upon the flux rope in the corona. This dynamic prominence was surrounded by a coronal cavity, with synthetic observations reproducing the relationship between cavity, prominence, and bright coronal flows within the cavity well (Fig. 20b).

Fan (2017) took a somewhat different approach, imposing a time-varying electric field at the lower boundary of a simulated quasi-steady coronal helmet streamer in a manner consistent with the kinematic emergence of magnetic twist. The MHD 
a Prominence density isocontours

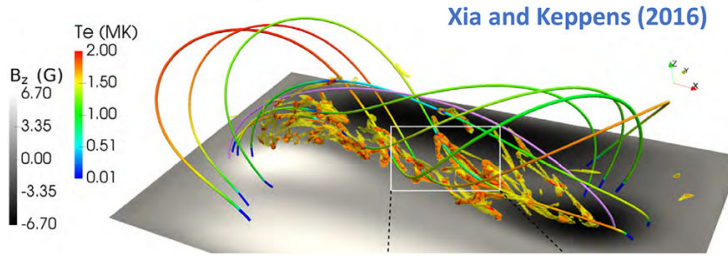

Xia and Keppens (2016)

C Prominence carrying field lines

Fan (2017)

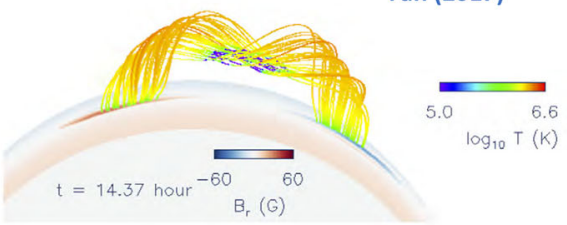

\section{b 171 Angstrom}

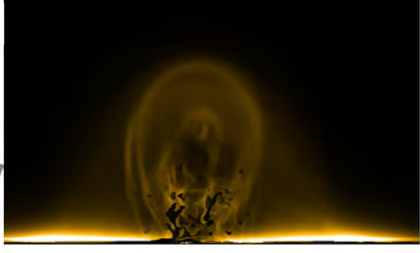

d White light

e 171 Angstrom

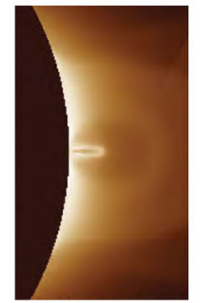

Fig. 20 Simulations in which prominences dynamically form within 3D magnetic structures (left) a Xia and Keppens (2016) and c Fan (2017) sample magnetic field lines with cool, dense prominence plasma supported in dips; (right) Synthetic observations of prominence material in absorption surrounded by a coronal cavity from b Xia and Keppens (2016) and d a simulation similar to Fan (2017) that was presented in Gibson et al. (2016). Images reproduced with permission, a-c copyright by AAS

equations were solved including empirically-defined coronal heating, optically-thin radiative cooling and electron heat conduction. The result was a confined magnetic flux rope in quasi-equilibrium beneath the streamer, within which a cold, dense prominence formed due to a runaway radiative instability (Fig. 20c). As the prominence formed, its mass dragged the magnetic field downward, leading to a significantly non-force free magnetic configuration.

These two simulations differed in their set-up and in some of their objectives: Xia and Keppens (2016) provided a high-resolution view of prominence fine-structure dynamics within a coronal magnetic configuration, while Fan (2017) studied the formation and evolution of the prominence in spherical coordinates, capturing its ultimate eruption into a background solar wind. It is interesting therefore that the two simulations obtained such similar-and observationally realistic_-prominence-cavity morphologies (Fig. 20b/d/e). They also demonstrated qualitatively similar dynamic processes controlling the prominence's condensation and ongoing evolution, in a manner extending upon but ultimatley consistent with previous 1D TNE simulations. Finally, both simulations noted significant departures from force-freeness as gravity dragged the magnetic field downward, in what was, however, still a relatively low- $\beta$ plasma with mass accumulating in magnetic dips.

\section{Conclusions}

Bearing in mind that our magnetic skeleton is neither completely rigid nor unchanging, let us consider what we have learned. If magnetic dips represent the bones of the prominence, then perhaps we may think of magnetic separatrices and flux surfaces 
as the joints and tissues connecting them. The challenge is then to arrange these structures in such a way that the dual requirements of consistency with observations and MHD force balance are satisfied. The topologies we have discussed-sheared arcade, flux rope, and spheromak - represent possible magnetic skeleton models that would achieve this. Based on the research reviewed here, we come to a few general conclusions:

(1) Both the sheared arcade and flux rope models are consistent with the basic observations of the prominence spine, barbs, filament channel, and counterstreaming flows. A spheromak configuration, although consistent with the prominence spine and chirality properties, is not consistent with most linear-polarization observations of coronal cavities (as discussed in Sect. 3.3).

(2) Some prominence-related observations strongly imply a flux rope model, including cavities with their nested toroidal structures and flows, well-defined (non-erupting) sigmoidal loops, and filaments/sigmoids that survive a partial eruption. It must be emphasized that such structures may exist in quasi-equilibrium for many hours or even days; however, ultimately they do tend to erupt. It is possible that there is a spectrum-from sheared arcade, to flux rope with BPSS, to flux rope with underlying X line- that may be directly associated with the stability of the prominence. It is also possible that the topological stage at which stability is lost differs for active region vs. quiescent prominences.

(3) Many of the assumptions that underlie the construction of a magnetic skeleton, including the necessity of dips and the force-free nature of the prominence and surrounding corona, should be examined in light of the full thermodynamics of the prominence-corona environment. The assumptions may hold to first order, but, in particular when comparing models to observations, a deeper level of understanding is needed beyond the bones of the skeleton. Numerical simulations are of particular use here, as they provide a means of simultaneously treating the global magnetic configuration and the thermodynamic properties of the prominence.

A final, personal reflection: It is clear that by selecting particular observations one can find evidence for any or all of the models/ magnetic topologies discussed in this review. This brings to mind the story of the elephant and three blind men, each of whom had dramatically different perspectives on what they were encountering. Ultimately, any model that fails to explain all of the observations is suspect; this motivates the hyperrealism of MHD thermodynamic simulations. I expect that future efforts are likely to move away from idealized models and towards data-driven simulations, with better and more comprehensive observations a necessary requirement for progress.

Despite all of the caveats presented in this review, I feel that the concept of a (dynamic) magnetic skeleton will be useful to such endeavors. The skeleton is a framework within which observations of the prominence and its coronal environment may be boiled down to essentials, so that a data-driven, 3D MHD-thermodynamics approach to modeling the prominence in all its complexity will be possible.

Acknowledgements I thank B. C. Low, Yuhong Fan, and Anna Malanushenko for many stimulating discussions. I also thank Lika Guhathakurta for being the first to introduce me to the "magnetic skeleton" metaphor. NCAR is supported by the National Science Foundation. This work was funded in part by NASA Grant NNX16AH02G. 
Open Access This article is distributed under the terms of the Creative Commons Attribution 4.0 International License (http://creativecommons.org/licenses/by/4.0/), which permits unrestricted use, distribution, and reproduction in any medium, provided you give appropriate credit to the original author(s) and the source, provide a link to the Creative Commons license, and indicate if changes were made.

\section{References}

Amari T, Aly JJ (1989) Interaction between a line current and a two-dimensional constant- $\alpha$ force-free field: an analytical model for quiescent prominences. Astron Astrophys 208:261-270

Amari T, Aly JJ (1990) Extended massive current sheet in a two-dimensional constant- $\alpha$ force-free field: a model for quiescent prominences I. Theory. Astron Astrophys 231:213-220

Amari T, Aly JJ (1992) Extended massive current sheet in a two-dimensional constant- $\alpha$ force-free field: a model for quiescent prominences. II. Existence and properties of inverse configurations. Astron Astrophys 265:791-805

Amari T, Démoulin P, Browning P, Hood A, Priest E (1991) The creation of the magnetic environment for prominence formation in a coronal arcade. Astron Astrophys 241:604-612

Amari T, Luciani JF, Mikić Z, Linker J (1999) Three-dimensional solutions of magnetohydrodynamic equations for prominence magnetic support: twisted magnetic flux rope. Astrophys J Lett 518:57. https://doi.org/10.1086/312053

Amari T, Luciani JF, Mikić Z, Linker J (2000) A twisted flux rope model for coronal mass ejections and two-ribbon flares. Astrophys J 529:L49. https://doi.org/10.1086/312444

Antiochos SK, Klimchuk JA (1991) A model for the formation of solar prominences. Astrophys J 378:372377. https://doi.org/10.1086/170437

Antiochos SK, Dahlburg RB, Klimchuk JA (1994) The magnetic field of solar prominences. Astrophys J Lett 420:41. https://doi.org/10.1086/187158

Antiochos SK, MacNeice PJ, Spicer DS, Klimchuk JA (1999) The dynamic formation of prominence condensations. Astrophys J 512:985. https://doi.org/10.1086/306804

Antiochos SK, MacNeice PJ, Spicer DS (2000) The thermal nonequilibrium of prominences. Astrophys J 536:494. https://doi.org/10.1086/308922

Anzer U (1969) Stability analysis of the Kippenhahn-Schlüter model of solar filaments. Solar Phys 8:37-52. https://doi.org/10.1007/BF00150656

Anzer U, Heinzel P (2007) Is the magnetic field in quiescent prominences force-free? Astron Astrophys 467:1285-1288. https://doi.org/10.1051/0004-6361:20066817

Arregui I, Oliver R, Ballester JL (2018) Prominence oscillations. Living Rev Sol Phys 15:3. https://doi.org/ $10.1007 / \mathrm{s} 41116-018-0012-6$

Aulanier G, Démoulin P (1998) 3-D magnetic configurations supporting prominences. I. The natural presence of lateral feet. Astron Astrophys 329:1125

Aulanier G, Démoulin P, van Driel-Gesztelyi L, Mein P, Deforest C (1998) 3-D magnetic configurations supporting prominences. II. The lateral feet as a perturbation of a twisted flux-tube. Astron Astrophys 335:309-322

Aulanier G, Démoulin P, Mein N, van Driel-Gesztelyi L, Mein P, Schmieder B (1999) 3-D magnetic configurations supporting prominences. III. Evolution of fine structures observed in a filament channel. Astron Astrophys 342:867-880

Aulanier G, DeVore CR, Antiochos SK (2002) Prominence magnetic dips in three-dimensional sheared arcades. Astrophys J Lett 567:L97-L101. https://doi.org/10.1086/339436

Aulanier G, Pariat E, Démoulin P (2005) Current sheet formation in quasi-separatrix layers and hyperbolic flux tubes. Astron Astrophys 444:961-976. https://doi.org/10.1051/0004-6361:20053600

Aulanier G, Török T, Démoulin P, DeLuca EE (2010) Formation of torus-unstable flux ropes and electric currents in erupting sigmoids. Astrophys J 708:314. https://doi.org/10.1088/0004-637X/708/1/314

Aurass H, Vršnak B, Hofmann A, Rudžjak V (1999) Flares in sigmoidal coronal structures_a case study. Solar Phys 190:267-293. https://doi.org/10.1023/A:1005261709955

Bąk-Stęślicka U, Gibson S, Chmielewska E (2016) Line-of-sight velocity as a tracer of coronal cavity magnetic structure. Front Astron Space Sci 3:7. https://doi.org/10.3389/fspas.2016.00007 
Bạk-Stęślicka U, Gibson SE, Fan Y, Bethge C, Forland B, Rachmeler LA (2013) Twisted magnetic structure of solar prominence cavities: new observational signature revealed by coronal magnetometry. Astrophys J 770:28. https://doi.org/10.1088/2041-8205/770/2/L28

Ballester JL (2015) Magnetism and dynamics of prominences: MHD waves. In: Engvold O, Vial JC (eds) Solar prominences, vol 415. Springer, Cham, pp 259-296. https://doi.org/10.1007/978-3-319-104164_11

Berger TE, Shine RA, Slater GL, Tarbell TD, Title AM, Okamoto TJ, Ichimoto K, Katsukawa Y, Suematsu Y, Tsuneta S, Lites BW, Shimizu T (2008) Hinode SOT observations of solar quiescent prominence dynamics. Astrophys J Lett 676:L89-L92. https://doi.org/10.1086/587171

Berger TE, Slater G, Hurlburt N, Shine R, Tarbell T, Title A, Lites BW, Okamoto TJ, Ichimoto K, Katsukawa Y, Magara T, Suematsu Y, Shimizu T (2010) Quiescent prominence dynamics observed with the Hinode solar optical telescope. I. Turbulent upflow plumes. Astrophys J 716:1288-1307. https://doi.org/10. 1088/0004-637X/716/2/1288

Berger T, Testa P, Hillier A, Boerner P, Low BC, Shibata K, Schrijver C, Tarbell T, Title A (2011) Magnetothermal convection in solar prominences. Nature 472:197-200. https://doi.org/10.1038/nature09925

Bommier V, Landi Degl'Innocenti E, Leroy JL, Sahal-Brechot S (1994) Complete determination of the magnetic field vector and of the electron density in 14 prominences from linear polarization measurements in the $\mathrm{HeI} \mathrm{D}_{3}$ and $\mathrm{H} \alpha$ lines. Solar Phys 154:231-260. https://doi.org/10.1007/BF00681098

Canfield RC, Hudson HS, McKenzie DE (1999) Sigmoidal morphology and eruptive solar activity. Geophys Res Lett 26:627. https://doi.org/10.1029/1999GL900105

Casini R, López Ariste A, Tomczyk S, Lites BW (2003) Magnetic maps of prominences from full stokes analysis of the He I D3 line. Astrophys J Lett 598:L67-L70. https://doi.org/10.1086/380496

Chae J (2010) Dynamics of vertical threads and descending knots in a hedgerow prominence. Astrophys J 714:618-629. https://doi.org/10.1088/0004-637X/714/1/618

Chae J, Moon YJ, Park YD (2005) The magnetic structure of filament barbs. Astrophys J 626:574-578. https://doi.org/10.1086/429797

Démoulin P (1998) Magnetic fields in filaments (review). In: Webb DF, Schmieder B, Rust DM (eds) IAU Colloquium 167: New perspectives on solar prominences. ASP conference series, vol 150. Astronomical Society of the Pacific, p 78

Démoulin P, Forbes TG (1992) Weighted current sheets supported in normal and inverse configurations: a model for prominence observations. Astrophys J 387:394-402. https://doi.org/10.1086/171091

Démoulin P, Priest ER (1993) A model for an inverse-polarity prominence supported in a dip of a quadrupolar region. Solar Phys 144:283-305. https://doi.org/10.1007/BF00627594

Démoulin P, Raadu MA, Malherbe JM (1992) Linear force-free magnetic field around quiescent solar prominences computed from observable boundary conditions. Astron Astrophys 257:278-286

Démoulin P, Henoux JC, Priest ER, Mandrini CH (1996) Quasi-separatrix layers in solar flares. I. Method. Astron Astrophys 308:643-655

DeVore CR, Antiochos SK (2000) Dynamical formation and stability of helical prominence magnetic fields. Astrophys J 539:954-963. https://doi.org/10.1086/309275

Dove J, Gibson S, Rachmeler LA, Tomczyk S, Judge P (2011) A ring of polarized light: evidence for twisted coronal magnetism in cavities. Astrophys J Lett 731:1. https://doi.org/10.1088/2041-8205/731/1/L1

Dudík J, Aulanier G, Schmieder B, Zapiór M, Heinzel P (2012) Magnetic topology of bubbles in quiescent prominences. Astrophys J 761:9. https://doi.org/10.1088/0004-637X/761/1/9

Engvold O (1989) Prominence environment. In: Priest ER (ed) Dynamics and structures of quiescent prominences. Astrophysics and space science library, vol 150. Kluwer Academic, Dordrecht, pp 4776. https://doi.org/10.1007/978-94-009-3077-3_3

Engvold O (2015) Description and classification of prominences. In: Engvold O, Vial JC (eds) Solar prominences, vol 415. Springer, Cham, pp 31-60. https://doi.org/10.1007/978-3-319-10416-4_2

Engvold O, Vial JC (eds) (2015) Solar prominences. Astrophysics and space science library. Springer, Cham. https://doi.org/10.1007/978-3-319-10416-4

Fan Y (2005) Coronal mass ejections as loss of confinement of kinked magnetic flux ropes. Astrophys J 630:543. https://doi.org/10.1086/431733

Fan Y (2012) Thermal signatures of tether-cutting reconnections in pre-eruption coronal flux ropes: hot central voids in coronal cavities. Astrophys J 758:60. https://doi.org/10.1088/0004-637X/758/1/60. arXiv: 1205.1028 
Fan Y (2015) MHD equilibria and triggers for eruption. In: Engvold O, Vial JC (eds) Solar prominences. Astrophysics and space science library, vol 415. Springer, Cham, pp 297-322. https://doi.org/10.1007/ 978-3-319-10416-4_12

Fan Y (2017) MHD simulations of the eruption of coronal flux ropes under coronal streamers. Astrophys J 844:26. https://doi.org/10.3847/1538-4357/aa7a56. arXiv:1706.06076

Fan Y, Gibson SE (2004) Numerical simulations of three-dimensional coronal magnetic fields resulting from the emergence of twisted magnetic flux tubes. Astrophys J 609:1123. https://doi.org/10.1086/ 421238

Fan Y, Gibson SE (2006) On the nature of the X-ray bright core in a stable filament channel. Astrophys J Lett 641:149. https://doi.org/10.1086/504107

Forbes TG, Isenberg PA (1991) A catastrophe mechanism for coronal mass ejections. Astrophys J 373:294307. https://doi.org/10.1086/170051

Forland BF, Gibson SE, Dove JB, Rachmeler LA, Fan Y (2013) Coronal cavity survey: morphological clues to eruptive magnetic topologies. Solar Phys 288:603-615. https://doi.org/10.1007/s11207-013-03611

Fuller J, Gibson SE, de Toma G, Fan Y (2008) Observing the unobservable? Modeling coronal cavity density. Astrophys J 678:515. https://doi.org/10.1086/533527

Galsgaard K, Titov VS, Neukirch T (2003) Magnetic pinching of hyperbolic flux tubes II. Dynamic numerical model. Astrophys J 595:506. https://doi.org/10.1086/377258

Gibson S (2014) Magnetism and the invisible man: the mysteries of coronal cavities. In: Schmieder B, Malherbe JM, Wu ST (eds) Nature of prominences and their role in space weather. IAU Symposium, vol 300. Cambridge University Press, Cambridge, pp 139-146. https://doi.org/10.1017/ S1743921313010879

Gibson S (2015) Coronal cavities: observations and implications for the magnetic environment of prominences. In: Engvold O, Vial JC (eds) Solar prominences. Astrophysics and space science library, vol 415. Springer, Cham, pp 323-353. https://doi.org/10.1007/978-3-319-10416-4_13

Gibson SE, Fan Y (2006a) Coronal prominence structure and dynamics: a magnetic flux rope interpretation. J Geophys Res 111:A12103. https://doi.org/10.1029/2006JA011871

Gibson SE, Fan Y (2006b) The partial expulsion of a magnetic flux rope. Astrophys J Lett 637:65. https:// doi.org/10.1086/500452

Gibson SE, Fan Y (2008) Partially-ejected flux ropes: implications for interplanetary coronal mass ejections. J Geophys Res 113:A09103. https://doi.org/10.1029/2008JA013151

Gibson SE, Low BC (1998) A time-dependent three-dimensional magnetohydrodynamic model of the coronal mass ejection. Astrophys J 493:460. https://doi.org/10.1086/305107

Gibson SE, Low BC (2000) Three-dimensional and twisted: an MHD interpretation of on-disk observational characteristics of coronal mass ejections. J Geophys Res 105(18):187. https://doi.org/10.1029/ 1999JA000317

Gibson SE, Fletcher L, Del Zanna G, Pike CD, Mason HE, Mandrini CH, Démoulin P, Gilbert H, Burkepile J, Holzer T, Alexander D, Liu Y, Nitta N, Qiu J, Schmieder B, Thompson BJ (2002) The structure and evolution of a sigmoidal active region. Astrophys J 574:265. https://doi.org/10.1086/341090

Gibson SE, Fan Y, Mandrini C, Fisher G, Démoulin P (2004) Observational consequences of a magnetic flux rope emerging into the corona. Astrophys J 617:600. https://doi.org/10.1086/425294

Gibson SE, Fan Y, Török T, Kliem B (2006a) The evolving sigmoid: evidence for magnetic flux ropes in the corona before, during, and after CMEs. In: Baker DN, Klecker B, Schwartz SJ, Schwenn R, Von Steiger R (eds) Solar dynamics and its effects on the heliosphere and earth. Space sciences series of ISSI, vol 22. Springer, New York, pp 131-144. https://doi.org/10.1007/978-0-387-69532-7_10

Gibson SE, Foster D, Burkepile J, de Toma G, Stanger A (2006b) The calm before the storm: the link between quiescent cavities and CMEs. Astrophys J 641:590. https://doi.org/10.1086/500446

Gibson SE, Kucera TA, Rastawicki D, Dove J, de Toma G, Hao J, Hill S, Hudson HS, Marque C, McIntosh PS, Rachmeler L, Reeves KK, Schmieder B, Schmit DJ, Seaton DB, Sterling AC, Tripathi D, Williams DR, Zhang M (2010) Three-dimensional morphology of a coronal prominence cavity. Astrophys J 723:1133. https://doi.org/10.1088/0004-637X/724/2/1133

Gibson S, Kucera T, White S, Dove J, Fan Y, Forland B, Rachmeler L, Downs C, Reeves K (2016) FORWARD: a toolset for multiwavelength coronal magnetometry. Front Astron Space Sci 3:8. https://doi. org/10.3389/fspas.2016.00008

Gilbert H (2015) Energy balance. In: Engvold O, Vial JC (eds) Solar prominences. Astrophysics and space science library, vol 415. Springer, Cham, pp 157-178. https://doi.org/10.1007/978-3-319-10416-4_7 
Gilbert HR, Holzer TE, Burkepile JT, Hundhausen AJ (2000) Active and eruptive prominences and their relationship to coronal mass ejections. Astrophys J 537:503. https://doi.org/10.1086/309030

Gilbert HR, Hansteen VH, Holzer TE (2002) Neutral atom diffusion in a partially ionized prominence plasma. Astrophys J 577:464-474. https://doi.org/10.1086/342165

Glover A, Ranns NDR, Harra LK, Culhane JL (2000) The onset and association of CMEs with sigmoidal active regions. Geophys Res Lett 27:2161. https://doi.org/10.1029/2000GL000018

Gopalswamy N (2015) The dynamics of eruptive prominences. In: Engvold O, Vial JC (eds) Solar prominences, vol 415. Springer, Cham, pp 381-410. https://doi.org/10.1007/978-3-319-10416-4_15

Green LM, Kliem B (2009) Flux rope formation preceding coronal mass ejection onset. Astrophys J Lett 700:L83. https://doi.org/10.1088/0004-637X/700/2/L83

Green LM, Kliem B, Török T, van Driel-Gesztelyi L, Attrill GDR (2007) Transient coronal sigmoids and rotating erupting flux ropes. Solar Phys 246:345. https://doi.org/10.1007/s11207-007-9061-z

Gunár S, Mackay DH (2015a) 3D whole-prominence fine structure modeling. Astrophys J 803:64. https:// doi.org/10.1088/0004-637X/803/2/64

Gunár S, Mackay DH (2015b) 3D whole-prominence fine structure modeling. II. Prominence evolution. Astrophys J 812:93. https://doi.org/10.1088/0004-637X/812/2/93

Gunár S, Mackay DH, Anzer U, Heinzel P (2013) Non-linear force-free magnetic dip models of quiescent prominence fine structures. Astron Astrophys 551:A3. https://doi.org/10.1051/0004-6361/201220597

Gunár S, Schwartz P, Dudík J, Schmieder B, Heinzel P, Jurčák J (2014) Magnetic field and radiative transfer modelling of a quiescent prominence. Astron Astrophys 567:A123. https://doi.org/10.1051/0004$6361 / 201322777$

Gunár S, Heinzel P, Mackay DH, Anzer U (2016) Quiescent prominences in the era of ALMA: simulated observations using the 3D whole-prominence fine structure model. Astrophys J 833:141. https://doi. org/10.3847/1538-4357/833/2/141

Habbal SR, Druckmueller M, Morgan H, Scholl I, Rusin V, Daw A, Johnson J, Arndt M (2010) Total solar eclipse observations of hot prominence shrouds. Astrophys J 719:1362. https://doi.org/10.1088/0004$637 X / 719 / 2 / 1362$

Heinzel P (2015) Radiative transfer in solar prominences. In: Engvold O, Vial JC (eds) Solar prominences. Astrophysics and space science library, vol 415. Springer, Cham, pp 103-130. https://doi.org/10.1007/ 978-3-319-10416-4_5

Heinzel P, Anzer U (2001) Prominence fine structures in a magnetic equilibrium: two-dimensional models with multilevel radiative transfer. Astron Astrophys 375:1082-1090. https://doi.org/10.1051/00046361:20010926

Heinzel P, Gunár S, Anzer U (2015) Fast approximate radiative transfer method for visualizing the fine structure of prominences in the hydrogen $\mathrm{H} \alpha$ line. Astron Astrophys 579:A16. https://doi.org/10. 1051/0004-6361/201525716

Hillier A (2018) The magnetic Rayleigh-Taylor instability in solar prominences. Rev Mod Plasma Phys 2:1. https://doi.org/10.1007/s41614-017-0013-2

Hillier A, van Ballegooijen A (2013) On the support of solar prominence material by the dips of a coronal flux tube. Astrophys J 766:126. https://doi.org/10.1088/0004-637X/766/2/126. arXiv:1303.4130

Hillier A, Isobe H, Shibata K, Berger T (2011) Numerical simulations of the magnetic Rayleigh-Taylor instability in the Kippenhahn-Schlüter prominence model. Astrophys J Lett 736:L1. https://doi.org/ 10.1088/2041-8205/736/1/L1. arXiv:1107.4882

Hillier A, Berger T, Isobe H, Shibata K (2012a) Numerical simulations of the magnetic Rayleigh-Taylor instability in the Kippenhahn-Schlüter prominence model. I. Formation of upflows. Astrophys J 746:120. https://doi.org/10.1088/0004-637X/746/2/120

Hillier A, Isobe H, Shibata K, Berger T (2012b) Numerical simulations of the magnetic Rayleigh-Taylor instability in the Kippenhahn-Schlüter prominence model. II. Reconnection-triggered downflows. Astrophys J 756:110. https://doi.org/10.1088/0004-637X/756/2/110. arXiv:1106.2613

Hudson HS, Acton LW, Harvey KA, McKenzie DM (1999) A stable filament cavity with a hot core. Astrophys J 513:83. https://doi.org/10.1086/311892

James AW, Green LM, Palmerio E, Valori G, Reid HAS, Baker D, Brooks DH, van Driel-Gesztelyi L, Kilpua EKJ (2017) On-disc observations of flux rope formation prior to its eruption. Solar Phys 292:71. https:// doi.org/10.1007/s11207-017-1093-4. arXiv:1703.10837

Karna N, Pesnell WD, Zhang J (2015a) Appearances and statistics of coronal cavities during the ascending phase of solar cycle 24. Astrophys J 810:123. https://doi.org/10.1088/0004-637X/810/2/123 
Karna N, Zhang J, Pesnell WD, Hess Webber SA (2015b) Study of the 3D geometric structure and temperature of a coronal cavity using the limb synoptic map method. Astrophys J 810:124. https://doi.org/ 10.1088/0004-637X/810/2/124

Karna N, Zhang J, Pesnell WD (2017) The formation and maintenance of the dominant southern polar crown cavity of cycle 24. Astrophys J 835:135. https://doi.org/10.3847/1538-4357/835/2/135

Karpen JT (2015) Plasma structure and dynamics. In: Engvold O, Vial JC (eds) Solar prominences, vol 415. Springer, Cham, pp 237-257. https://doi.org/10.1007/978-3-319-10416-4_10

Karpen JT, Antiochos SK, Hohensee M, Klimchuk JA, MacNeice PJ (2001) Are magnetic dips necessary for prominence formation? Astrophys J Lett 553:L85-L88. https://doi.org/10.1086/320497

Khomenko E, Díaz A, de Vicente A, Collados M, Luna M (2014) Rayleigh-Taylor instability in prominences from numerical simulations including partial ionization effects. Astron Astrophys 565:A45. https:// doi.org/10.1051/0004-6361/201322918. arXiv:1403.4530

Kippenhahn R, Schlüter A (1957) Eine Theorie der solaren Filamente. Z Astrophys 43:36

Kliem B, Titov VS, Török T (2004) Formation of current sheets and sigmoidal structure by the kink instability of a magnetic loop. Astron Astrophys 413:L23-L26. https://doi.org/10.1051/0004-6361: 20031690. arXiv:astro-ph/0311199

Klimchuk JA (1990) Shear-induced inflation of coronal magnetic fields. Astrophys J 354:745-754. https:// doi.org/10.1086/168731

Koutchmy S, Baudin F, Bocchialini K, Daniel JY, Delaboudinière JP, Golub L, Lamy P, Adjabshirizadeh A (2004) The August 11th, 1999 CME. Astron Astrophys 420:709-718. https://doi.org/10.1051/00046361:20040109

Kucera TA (2015) Derivations and observations of prominence bulk motions and mass. In: Engvold O, Vial JC (eds) Solar prominences. Astrophysics and space science library, vol 415. Springer, Cham, pp 79-101. https://doi.org/10.1007/978-3-319-10416-4_4

Kucera TA, Gibson SE, Schmit DJ, Landi E, Tripathi D (2012) Temperature and EUV intensity in a coronal prominence cavity. Astrophys J 757:73. https://doi.org/10.1088/0004-637X/757/1/73

Kuckein C, Centeno R, Martínez Pillet V, Casini R, Manso Sainz R, Shimizu T (2009) Magnetic field strength of active region filaments. Astron Astrophys 501:1113-1121. https://doi.org/10.1051/00046361/200911800. arXiv:0904.4876

Kundu MR, Fuerst E, Hirth W, Butz M (1978) Multifrequency observations of solar filaments at centimeter wavelengths. Astron Astrophys 62:431-437

Kuperus M, Raadu MA (1974) The support of prominences formed in neutral sheets. Astron Astrophys $31: 189$

Kusano K (2005) Simulation study of the formation mechanism of sigmoidal structure in the solar corona. Astrophys J 631:1260-1269. https://doi.org/10.1086/432570

Labrosse N (2015) Derivation of the major properties of prominences using NLTE modeling. In: Engvold O, Vial JC (eds) Solar prominences. Astrophysics and space science library, vol 415. Springer, Cham, pp 131-155. https://doi.org/10.1007/978-3-319-10416-4_6

Labrosse N, Heinzel P, Vial JC, Kucera T, Parenti S, Gunár S, Schmieder B, Kilper G (2010) Physics of solar prominences: I-spectral diagnostics and non-LTE modelling. Space Sci Rev 151:243-332. https:// doi.org/10.1007/s11214-010-9630-6. arXiv:1001.1620

Lerche I, Low BC (1980) On the equilibrium of a cylindrical plasma supported horizontally by magnetic fields in uniform gravity. Solar Phys 67:229-243. https://doi.org/10.1007/BF00149804

Leroy JL (1977) On the intensity of magnetic field in quiescent prominences. Astron Astrophys 60:79-84

Leroy JL (1978) On the orientation of magnetic fields in quiescent prominences. Astron Astrophys 64:247252

Leroy JL (1988) The fine structure of prominences magnetic field: can we provide useful observational constraints? In: Altrock RC (ed) Solar and stellar coronal structure and dynamics. National Solar Observatory, New Mexico, pp 422-443

Leroy JL (1989) Observation of prominence magnetic fields. In: Priest ER (ed) Dynamics and structure of quiescent solar prominences. Astrophysics and space science library, vol 150. Kluwer Academic, Dordrecht, pp 77-113. https://doi.org/10.1007/978-94-009-3077-3_4

Leroy JL, Bommier V, Sahal-Brechot S (1984) New data on the magnetic structure of quiescent prominences. Astron Astrophys 131:33

Levens PJ, Schmieder B, Labrosse N, López Ariste A (2016) Structure of prominence legs: plasma and magnetic field. Astrophys J 818:31. https://doi.org/10.3847/0004-637X/818/1/31. arXiv:1512.04727 
Li X, Morgan H, Leonard D, Jeska L (2012) A solar tornado observed by AIA/SDO: rotational flow and evolution of magnetic helicity in a prominence and cavity. Astrophys J Lett 752:L22. https://doi.org/ 10.1088/2041-8205/752/2/L22

Linker J, Mikić Z, Lionello R, Riley P, Amari T, Odstrcil D (2003) Flux cancellation and coronal mass ejections. Phys Plasmas 10:1971-1978. https://doi.org/10.1063/1.1563668

Lionello R, Mikić Z, Linker JA, Amari T (2002) Magnetic field topology in prominences. Astrophys J 581:718-725. https://doi.org/10.1086/344222

Lites BW, Low BC (1997) Flux emergence and prominences: a new scenario for 3-dimensional field geometry based on observations with the advanced stokes polarimeter. Solar Phys 174:91. https://doi. org/10.1023/A:1004936204808

López Ariste A (2015) Magnetometry of prominences. In: Engvold O, Vial JC (eds) Solar prominences, vol 415. Springer, Cham, pp 179-203. https://doi.org/10.1007/978-3-319-10416-4_8

Low BC (1982) The vertical filamentary structures of quiescent prominences. Solar Phys 75:119-131. https://doi.org/10.1007/BF00153465

Low BC (1993) Force-free magnetic fields with singular current-density surfaces. Astrophys J 409:798-808. https://doi.org/10.1086/172710

Low BC (1994) Magnetohydrodynamic processes in the solar corona: flares, coronal mass ejections, and magnetic helicity. Phys Plasmas 1:1684-1690. https://doi.org/10.1063/1.870671

Low BC (1996) Solar activity and the corona. Solar Phys 167:217. https://doi.org/10.1007/BF00146338

Low BC (2001) Coronal mass ejections, magnetic flux ropes, and solar magnetism. J Geophys Res 106:25141-25164. https://doi.org/10.1029/2000JA004015

Low BC (2018) Coronal magnetism as a universal phenomenon. In: Engvold O, Skumanich A, Vial JC (eds) The Sun: a guide to stellar physics. Elsevier, Amsterdam

Low BC, Berger M (2003) A morphological study of helical coronal magnetic structures. Astrophys J 589:644. https://doi.org/10.1086/374614

Low BC, Egan AK (2014) Steady fall of isothermal, resistive-viscous, compressible fluid across magnetic field. Phys Plasmas 21(6):062105. https://doi.org/10.1063/1.4882676

Low BC, Hundhausen JR (1995) Magnetostatic structures of the solar corona. II. The magnetic topology of quiescent prominences. Astrophys J 443:818. https://doi.org/10.1086/175572

Low BC, Petrie GJD (2005) The internal structures and dynamics of solar quiescent prominences. Astrophys J 626:551. https://doi.org/10.1086/430046

Low BC, Zhang M (2004) Magnetostatic structures of the solar corona. III. Normal and inverse quiescent prominences. Astrophys J 609:1098-1111. https://doi.org/10.1086/421256

Low BC, Liu W, Berger T, Casini R (2012) The hydromagnetic interior of a solar quiescent prominence. II. Magnetic discontinuities and cross-field mass transport. Astrophys J 757:21. https://doi.org/10.1088/ 0004-637X/757/1/21

Lugaz N (2015) Eruptive prominences and their impact on the earth and our life. In: Engvold O, Vial JC (eds) Solar prominences, vol 415. Springer, Cham, pp 433-453. https://doi.org/10.1007/978-3-31910416-4_17

Luna M, Karpen JT, DeVore CR (2012) Formation and evolution of a multi-threaded solar prominence. Astrophys J 746:30. https://doi.org/10.1088/0004-637X/746/1/30. arXiv:1201.3559

Luna M, Karpen J, Ballester JL, Muglach K, Terradas J, Kucera T, Gilbert H (2018) GONG catalog of solar filament oscillations near solar maximum. Astrophys J Suppl Ser 236:35. https://doi.org/10.3847/ 1538-4365/aabde7. arXiv:1804.03743

Mackay DH (2015) Formation and large-scale patterns of filament channels and filaments. In: Engvold O, Vial JC (eds) Solar prominences. Astrophysics and space science library, vol 415. Springer, Cham, pp 355-380. https://doi.org/10.1007/978-3-319-10416-4_14

Mackay DH, van Ballegooijen AA (2009) A non-linear force-free field model for the evolving magnetic structure of solar filaments. Solar Phys 260:321-346. https://doi.org/10.1007/s11207-009-9468-9

Mackay DH, Gaizauskas V, Yeates AR (2008) Where do solar filaments form? Consequences for theoretical models. Solar Phys 248:51-65. https://doi.org/10.1007/s11207-008-9127-6

Mackay DH, Karpen JT, Ballester JL, Schmieder B, Aulanier G (2010) Physics of solar prominences: IImagnetic structure and dynamics. Space Sci Rev 151:333-399. https://doi.org/10.1007/s11214-0109628-0. arXiv:1001.1635

Malanushenko A, Schrijver CJ, DeRosa ML, Wheatland MS (2014) Using coronal loops to reconstruct the magnetic field of an active region before and after a major flare. Astrophys J 783:102. https://doi.org/ 10.1088/0004-637X/783/2/102. arXiv:1312.5389 
Malherbe JM, Priest ER (1983) Current sheet models for solar prominences. I. Magnetohydrostatics of support and evolution through quasi-static models. Astron Astrophys 123:80-88

Maričić D, Vršnak B, Roša D (2009) Relative kinematics of the leading edge and the prominence in coronal mass ejections. Solar Phys 260:177. https://doi.org/10.1007/s11207-009-9421-y

Marqué C (2004) Radiometric observations of quiescent filament cavities. Astrophys J 602:1037. https:// doi.org/10.1086/381085

Martin SF (1998a) Conditions for the formation and maintenance of filaments (invited review). Solar Phys 182:107-137. https://doi.org/10.1023/A:1005026814076

Martin SF (1998b) Filament chirality: a link between fine-scale and global patterns (review). In: Webb DF, Schmieder B, Rust DM (eds) IAU Colloquium 167: New perspectives on solar prominences. ASP conference series, vol 150. Astronomical Society of the Pacific, San Francisco, p 419

Martin SF (2015) The magnetic field structure of prominences from direct and indirect observations. In: Engvold O, Vial JC (eds) Solar prominences, vol 415. Springer, Cham, pp 205-235. https://doi.org/ 10.1007/978-3-319-10416-4_9

Martin SF, McAllister AH (1996) The skew of X-ray coronal loops overlying H $\alpha$ filaments. In: Uchida Y, Kosugi T, Hudson HS (eds) IAU Colloquium 153: Magnetodynamic phenomena in the solar atmosphere. Prototypes of stellar magnetic activity. Springer, Dordrecht, pp 497-498. https://doi.org/10. 1007/978-94-009-0315-9_112

Martínez González MJ, Asensio Ramos A, Arregui I, Collados M, Beck C, de la Cruz Rodríguez J (2016) On the magnetism and dynamics of prominence legs hosting tornadoes. Astrophys J 825:119. https:// doi.org/10.3847/0004-637X/825/2/119. arXiv:1605.01183

McIntosh PS, Krieger AS, Nolte JT, Vaiana G (1976) Association of X-ray arches with chromospheric neutral lines. Solar Phys 49:57-77. https://doi.org/10.1007/BF00221485

Mercier C, Heyvaerts J (1977) The downward motions in quiescent prominences. Astron Astrophys 61:685693

Moore RL, Sterling AC, Hudson HS, Lemen JR (2001) Onset of the magnetic explosion in solar flares and coronal mass ejections. Astrophys J 552:833. https://doi.org/10.1086/320559

Nindos A, Patsourakos S, Vourlidas A, Tagikas C (2015) How common are hot magnetic flux ropes in the low solar corona? A statistical study of EUV observations. Astrophys J 808:117. https://doi.org/10. 1088/0004-637X/808/2/117. arXiv:1507.03766

Okamoto TJ, Liu W, Tsuneta S (2016) Helical motions of fine-structure prominence threads observed by Hinode and IRIS. Astrophys J 831:126. https://doi.org/10.3847/0004-637X/831/2/126. arXiv:1608.00123

Orozco Suárez D, Asensio Ramos A, Trujillo Bueno J (2012) Evidence for rotational motions in the feet of a quiescent solar prominence. Astrophys J Lett 761:L25. https://doi.org/10.1088/2041-8205/761/ 2/L25. arXiv:1211.6980

Panasenco O, Martin SF, Velli M (2014) Apparent solar tornado-like prominences. Solar Phys 289:603-622. https://doi.org/10.1007/s11207-013-0337-1. arXiv:1307.2303

Panesar NK, Innes DE, Tiwari SK, Low BC (2013) A solar tornado triggered by flares? Astron Astrophys 549:A105. https://doi.org/10.1051/0004-6361/201220503. arXiv:1211.6569

Parenti S (2014) Solar prominences: observations. Living Rev Sol Phys 11:1. https://doi.org/10.12942/lrsp2014- 1

Parenti S (2015) Spectral diagnostics of cool prominence and PCTR optically thin plasmas. In: Engvold O, Vial JC (eds) Solar prominences. Astrophysics and space science library, vol 415. Springer, Cham, pp 61-77. https://doi.org/10.1007/978-3-319-10416-4_3

Patsourakos S, Vourlidas A, Kliem B (2010a) Toward understanding the early stages of an impulsively accelerated coronal mass ejection. SECCHI observations. Astron Astrophys 522:A100. https://doi. org/10.1051/0004-6361/200913599. arXiv:1008.1171

Patsourakos S, Vourlidas A, Stenborg G (2010b) The genesis of an impulsive coronal mass ejection observed at ultra-high cadence by AIA on SDO. Astrophys J Lett 724:L188-L193. https://doi.org/10.1088/ 2041-8205/724/2/L188. arXiv:1010.5234

Patsourakos S, Vourlidas A, Stenborg G (2013) Direct evidence for a fast coronal mass ejection driven by the prior formation and subsequent destabilization of a magnetic flux rope. Astrophys J 764:125. https://doi.org/10.1088/0004-637X/764/2/125. arXiv:1211.7211

Petrie GJD, Low BC (2005) The dynamical consequences of spontaneous current sheets in quiescent prominences. Astrophys J Suppl Ser 159:288. https://doi.org/10.1086/431149

Pettit E (1932) Characteristic features of solar prominences. Astrophys J 76:9. https://doi.org/10.1086/ 143396 
Pevtsov AA (2002) Active-region filaments and X-ray sigmoids. Solar Phys 207:111-123. https://doi.org/ 10.1023/A:1015589802234

Pevtsov AA, Canfield RC, McClymont AN (1997) On the subphotospheric origin of coronal electric currents. Astrophys J 481:973. https://doi.org/10.1086/304065

Pneuman GW (1983) The formation of solar prominences by magnetic reconnection and condensation. Solar Phys 88:219. https://doi.org/10.1007/BF00196189

Priest ER, Démoulin P (1995) Three-dimensional magnetic reconnection without null points. 1. Basic theory of magnetic flipping. J Geophys Res 100:23,443-23,464. https://doi.org/10.1029/95JA02740

Priest ER, Hood AW, Anzer U (1989) A twisted flux-tube model for solar prominences. I. General properties. Astrophys J 344:1010-1025. https://doi.org/10.1086/167868

Rachmeler LA, Gibson SE, Dove JB, DeVore CR, Fan Y (2013) Polarimetric properties of flux ropes and sheared arcades in coronal prominence cavities. Solar Phys 288:617-636. https://doi.org/10.1007/ s11207-013-0325-5. arXiv:1304.7594

Reeves KK, Gibson SE, Kucera TA, Hudson HS (2012) Thermal properties of coronal cavities observed with the X-ray telescope on Hinode. Astrophys J 746:146. https://doi.org/10.1088/0004-637X/746/ 2/146

Régnier S, Walsh RW, Alexander CE (2011) A new look at a polar crown cavity as observed by SDO/AIA. Structure and dynamics. Astron Astrophys 533:L1. https://doi.org/10.1051/0004-6361/201117381. arXiv:1107.3451

Ridgway C, Priest ER, Amari T (1991) A twisted flux tube model for solar prominences. III. Magnetic support. Astrophys J 367:321-332. https://doi.org/10.1086/169631

Rust DM (1967) Magnetic fields in quiescent solar prominences. I. Observations. Astrophys J 150:313. https://doi.org/10.1086/149333

Rust DM, Kumar A (1994) Helical magnetic field in filaments. Solar Phys 155:69. https://doi.org/10.1007/ BF00670732

Rust DM, Kumar A (1996) Evidence for helically kinked magnetic flux ropes in solar eruptions. Astrophys J Lett 464:L199. https://doi.org/10.1086/310118

Ryutova M, Berger T, Frank Z, Tarbell T, Title A (2010) Observation of plasma instabilities in quiescent prominences. Solar Phys 267:75-94. https://doi.org/10.1007/s11207-010-9638-9

Saito K, Hyder CL (1968) A concentric ellipse multiple-arch system in the solar corona. Solar Phys 5:61. https://doi.org/10.1007/BF00147121

Saito K, Tandberg-Hanssen E (1973) The arch systems, cavities, and prominences in the helmet streamer observed at the solar eclipse, November 12, 1966. Solar Phys 31:105. https://doi.org/10.1007/ BF00156076

Savcheva A, van Ballegooijen A (2009) Nonlinear force-free modeling of a long-lasting coronal sigmoid. Astrophys J 703:1766. https://doi.org/10.1088/0004-637X/703/2/1766

Savcheva A, Green LM, van Ballegooijen AA, DeLuca EE (2012a) Photospheric flux cancellation and the build-up of sigmoidal flux ropes on the Sun. Astrophys J 759:105. https://doi.org/10.1088/0004$637 \mathrm{X} / 759 / 2 / 105$

Savcheva A, Pariat E, van Ballegooijen A, Aulanier G, DeLuca E (2012b) Sigmoidal active region on the sun: comparison of a magnetohydrodynamical simulation and a nonlinear force-free field model. Astrophys J 750:15. https://doi.org/10.1088/0004-637X/750/1/15

Savcheva A, Van Ballegooijen A, DeLuca E (2012c) Field topology analysis of a long-lasting coronal sigmoid. Astrophys J 744:78. https://doi.org/10.1088/0004-637X/744/1/78

Schmahl EJ (1979) The prominence-corona interface-a review. In: Jensen E, Maltby P, Orrall FQ (eds) IAU Colloquium 44: Physics of solar prominences, pp 102-120

Schmieder B, Raadu MA, Wiik JE (1991) Fine structure of solar filaments. II. Dynamics of threads and footpoints. Astron Astrophys 252:353-365

Schmieder B, Chandra R, Berlicki A, Mein P (2010) Velocity vectors of a quiescent prominence observed by Hinode/SOT and the MSDP (Meudon). Astron Astrophys 514:A68. https://doi.org/10.1051/00046361/200913477. arXiv:0911.5091

Schmieder B, Mein P, Mein N, Levens PJ, Labrosse N, Ofman L (2017) H $\alpha$ Doppler shifts in a tornado in the solar corona. Astron Astrophys 597:A109. https://doi.org/10.1051/0004-6361/201628771. arXiv: 1612.02232

Schmit DJ, Gibson SE (2011) Forward modeling cavity density: a multi-instrument diagnostic. Astrophys J 733:1. https://doi.org/10.1088/0004-637X/733/1/1 
Schmit DJ, Gibson S (2013) Diagnosing the prominence-cavity connection. Astrophys J 770:35. https:// doi.org/10.1088/0004-637X/770/1/35. arXiv:1304.7595

Schmit DJ, Gibson SE (2014) The formation of a cavity in a 3D flux rope. Proc Int Astron Union 8:147-150. https://doi.org/10.1017/S1743921313010880. arXiv:1311.2384

Schmit DJ, Gibson SE, Tomczyk S, Reeves KK, Sterling AC, Brooks DH, Williams DR, Tripathi D (2009) Large-scale flows in prominence cavities. Astrophys J Lett 700:96. https://doi.org/10.1088/0004637X/700/2/L96

Schmit DJ, Gibson S, Luna M, Karpen J, Innes D (2013) Prominence mass supply and the cavity. Astrophys J 779:156. https://doi.org/10.1088/0004-637X/779/2/156. arXiv:1311.2382

Schonfelder AO, Hood AW (1995) Current sheet model for inverse polarity prominences in twisted flux tubes. Solar Phys 157:223-249. https://doi.org/10.1007/BF00680619

Serio S, Vaiana GS, Godoli G, Motta S, Pirronello V, Zappala RA (1978) Configuration and gradual dynamics of prominence-related X-ray coronal cavities. Solar Phys 59:65-86. https://doi.org/10.1007/ BF00154932

Straka RM, Papagiannis MD, Kogut JA (1975) Study of a filament with a circularly polarized beam at 3.8 cm. Solar Phys 45:131. https://doi.org/10.1007/BF00152225

Su Y, van Ballegooijen A (2012) Observations and magnetic field modeling of a solar polar crown prominence. Astrophys J 757:168. https://doi.org/10.1088/0004-637X/757/2/168. arXiv:1208.1524

Su Y, Gömöry P, Veronig A, Temmer M, Wang T, Vanninathan K, Gan W, Li Y (2014) Solar magnetized tornadoes: rotational motion in a tornado-like prominence. Astrophys J Lett 785:L2. https://doi.org/ 10.1088/2041-8205/785/1/L2. arXiv:1312.5226

Tandberg-Hanssen E (1974) Solar prominences. Geophysics and astrophysics monographs, vol 12. D. Reidel, Dordrecht. https://doi.org/10.1007/978-94-010-2136-4

Tandberg-Hanssen E (1995) The nature of solar prominences. Astrophysics and space science library, vol 199. Kluwer Academic, Dordrecht. https://doi.org/10.1007/978-94-017-3396-0

Tang F (1987) Quiescent prominences—where are they formed? Solar Phys 107:233. https://doi.org/10. 1007/BF00152021

Terradas J, Soler R, Luna M, Oliver R, Ballester JL (2015) Morphology and dynamics of solar prominences from 3D MHD simulations. Astrophys J 799:94. https://doi.org/10.1088/0004-637X/799/1/ 94. arXiv: 1412.7438

Terradas J, Soler R, Luna M, Oliver R, Ballester JL, Wright AN (2016) Solar prominences embedded in flux ropes: morphological features and dynamics from 3D MHD simulations. Astrophys J 820:125. https://doi.org/10.3847/0004-637X/820/2/125. arXiv:1512.07096

Titov VS, Démoulin P (1999) Basic topology of twisted magnetic configurations in solar flares. Astron Astrophys 351:707

Titov VS, Hornig G, Démoulin P (2002) Theory of magnetic connectivity in the solar corona. J Geophys Res 107:1164. https://doi.org/10.1029/2001JA000278

Titov VS, Galsgaard K, Neukirch T (2003) Magnetic pinching of hyperbolic flux tubes. I. Basic estimations. Astrophys J 582:1172. https://doi.org/10.1086/344799

Tomczyk S, Card GL, Darnell T, Elmore DF, Lull R, Nelson PG, Streander KV, Burkepile J, Casini R, Judge PG (2008) An instrument to measure coronal emission line polarization. Solar Phys 247:411. https:// doi.org/10.1007/s11207-007-9103-6

Török T, Kliem B (2005) Confined and ejective eruptions of kink-unstable flux ropes. Astrophys J Lett 630:L97

Török T, Kliem B, Titov VS (2004) Ideal kink instability of a magnetic loop equilibrium. Astron Astrophys 413:L27

Tripathi D, Gibson SE, Qiu J, Fletcher L, Liu R, Gilbert H, Mason HE (2009) Partially-erupting prominences: a comparison between observations and model-predicted observables. Astron Astrophys 498:295-305. https://doi.org/10.1051/0004-6361/200809801. arXiv:0902.1228

Vaiana GS, Krieger AS, Timothy AF (1973) Identification and analysis of structures in the corona from X-ray photography. Solar Phys 32:81-116. https://doi.org/10.1007/BF00152731

van Ballegooijen AA (2004) Observations and modeling of a filament on the sun. Astrophys J 612:519. https://doi.org/10.1086/422512

van Ballegooijen AA, Cranmer SR (2010) Tangled magnetic fields in solar prominences. Astrophys J 711:164. https://doi.org/10.1088/0004-637X/711/1/164

van Ballegooijen AA, Mackay DH (2007) Model for the coupled evolution of subsurface and coronal magnetic fields in solar active regions. Astrophys J 659:1713-1725. https://doi.org/10.1086/512849 
van Ballegooijen AA, Martens PCH (1999) Formation and eruption of solar prominences. Astrophys J 343:971

Vršnak B, Maričić D, Stanger AL, Veronig A (2004) Coronal mass ejection of 15 May 2001: II. Coupling of the CME acceleration and the flare energy release. Solar Phys 225:355-378. https://doi.org/10.1007/ s11207-004-4995-x

Waldmeier M (1970) The structure of the monochromatic corona in the surroundings of prominences. Solar Phys 15:167-175. https://doi.org/10.1007/BF00149483

Wang YM, Stenborg G (2010) Spinning motions in coronal cavities. Astrophys J Lett 719:181. https://doi. org/10.1088/2041-8205/719/2/L181

Warmuth A, Mann G (2005) A model of the Alfvén speed in the solar corona. Astron Astrophys 435:11231135. https://doi.org/10.1051/0004-6361:20042169

Webb D (2015) Eruptive prominences and their association with coronal mass ejections. In: Engvold O, Vial JC (eds) Solar prominences, vol 415. Springer, Cham, pp 411-432. https://doi.org/10.1007/9783-319-10416-4_17

Wedemeyer S, Scullion E, Rouppe van der Voort L, Bosnjak A, Antolin P (2013) Are giant tornadoes the legs of solar prominences? Astrophys J 774:123. https://doi.org/10.1088/0004-637X/774/2/123. arXiv: 1306.2661

Welsch BT, DeVore CR, Antiochos SK (2005) Magnetic reconnection models of prominence formation. Astrophys J 634:1395-1404. https://doi.org/10.1086/491641

Williams DR, Harra LK, Brooks DH, Imada S, Hansteen VH (2009) Evidence from the extreme-ultraviolet imaging spectrometer for axial filament rotation before a large flare. Publ Astron Soc Japan 61:493497. https://doi.org/10.1093/pasj/61.3.493

Xia C, Keppens R (2016) Formation and plasma circulation of solar prominences. Astrophys J 823:22. https://doi.org/10.3847/0004-637X/823/1/22. arXiv:1603.05397

Yurchyshyn VB (2002) Evidence of a flux-rope model for corona mass ejections based on observations of the limb prominence eruption on 2002 January 4. Astrophys J 576:493-496. https://doi.org/10.1086/ 341628

Zhang J, Cheng X, Ding MD (2012) Observation of an evolving magnetic flux rope before and during a solar eruption. Nature Commun 3:747. https://doi.org/10.1038/ncomms1753. arXiv:1203.4859

Zirker JB, Engvold O, Martin SF (1998) Counter-streaming gas flows in solar prominences as evidence for vertical magnetic fields. Science 396:440

Publisher's Note Springer Nature remains neutral with regard to jurisdictional claims in published maps and institutional affiliations. 